\title{
The inflammatory cytokine IL-6 induces FRA1 deacetylation promoting colorectal cancer stem-like properties
}

\author{
Tingyang Wang ${ }^{1,2} \cdot$ Ping Song ${ }^{1,2} \cdot$ Tingting Zhong ${ }^{1,8} \cdot$ Xianjun Wang $^{1} \cdot$ Xueping Xiang $^{1,2} \cdot$ Qian Liu ${ }^{1} \cdot$ Haiyi Chen $^{3} \cdot$ \\ Tian $\mathrm{Xia}^{1} \cdot$ Hong $\mathrm{Liu}^{1,9} \cdot$ Yumiao $\mathrm{Niu}^{1} \cdot$ Yanshi Hu $\mathbb{D}^{4} \cdot$ Lei $\mathrm{Xu}^{5} \cdot$ Yingkuan Shao $\mathbb{D}^{1,2} \cdot$ Lijun Zhu ${ }^{6} \cdot$ Hongyan $\mathrm{Qi}^{1}$. \\ Jing Shen ${ }^{1} \cdot$ Tingjun Hou ${ }^{3,5} \cdot$ Riccardo Fodde $\mathbb{C}^{7} \cdot \operatorname{Jimin}$ Shao $^{1,2}$
}

Received: 18 October 2018 / Revised: 24 January 2019 / Accepted: 11 February 2019 / Published online: 25 February 2019

(c) The Author(s) 2019. This article is published with open access

\begin{abstract}
Colorectal cancer (CRC) has long been known for its tight association with chronic inflammation, thought to play a key role in tumor onset and malignant progression through the modulation of cancer stemness. However, the underlying molecular and cellular mechanisms are still largely elusive. Here we show that the IL-6/STAT3 inflammatory signaling axis induces the deacetylation of FRA1 at the Lys-116 residue located within its DNA-binding domain. The HDAC6 deacetylase underlies this key modification leading to the increase of FRA1 transcriptional activity, the subsequent transactivation of NANOG expression, and the acquisition of stem-like cellular features. As validated in a large $(n=123)$ CRC cohort, IL- 6 secretion was invariably accompanied by increased FRA1 deacetylation at $\mathrm{K} 116$ and an overall increase in its protein levels, coincident with malignant progression and poor prognosis. Of note, combined treatment with the conventional cytotoxic drug 5-FU together with Tubastatin A, a HDAC6-specific inhibitor, resulted in a significant in vivo synergistic inhibitory effect on tumor growth through suppression of CRC stemness. Our results reveal a novel transcriptional and posttranslational regulatory cross-talk between inflammation and stemness signaling pathways that underlie self-renewal and maintenance of CRC stem cells and promote their malignant behavior. Combinatorial treatment aimed at the core regulatory mechanisms downstream of IL-6 may offer a novel promising approach for CRC treatment.
\end{abstract}

Supplementary information The online version of this article (https:// doi.org/10.1038/s41388-019-0763-0) contains supplementary material, which is available to authorized users.

\section{Riccardo Fodde \\ r.fodde@erasmusmc.nl \\ $\triangle$ Jimin Shao \\ shaojimin@zju.edu.cn}

1 Department of Pathology \& Pathophysiology, and Cancer Institute of the Second Affiliated Hospital, Zhejiang University School of Medicine, Hangzhou, China

2 Key Laboratory of Disease Proteomics of Zhejiang Province, Key Laboratory of Cancer Prevention and Intervention of China National Ministry of Education, and Research Center for Air Pollution and Health, Zhejiang University School of Medicine, Hangzhou, China

3 College of Pharmaceutical Sciences, Zhejiang University, Hangzhou, China

4 Department of Bioinformatics, College of Life Sciences, Zhejiang

\section{Introduction}

Tumor-promoting inflammation is emerging as one of the main hallmarks of cancer [1] and colorectal cancer (CRC) best exemplifies the tight link between inflammation, tumor

University, Hangzhou, China

5 Institute of Bioinformatics and Medical Engineering, School of Electrical and Information Engineering, Jiangsu University of Technology, Changzhou, China

6 Key Laboratory of Precision Diagnosis and Treatment for Hepatobiliary and Pancreatic Tumor of Zhejiang Province, First Affiliated Hospital, Zhejiang University School of Medicine, Hangzhou, China

7 Department of Pathology, Erasmus MC Cancer Institute, Erasmus University Medical Center, Rotterdam, The Netherlands

8 Present address: Department of Pathology, Sir Run Run Shaw Hospital, Zhejiang University School of Medicine, Hangzhou, China

9 Present address: Zhejiang Normal University-Jinhua People's Hospital Joint Center for Biomedical Research, Jinhua, China 
onset, and malignant progression [2]. We and others have previously shown that interleukin-6 (IL-6) represents a key inflammatory cytokine and that its downstream effector STAT3 (signal transducer and activator of transcription 3) underlies CRC proliferation, epithelial-to-mesenchymal transition (EMT) [3], tumorigenesis [4], and stemness [5]. In hepatocellular carcinoma (HCC), tumor-associated macrophages promote $\mathrm{CD} 44^{+}$stem-like cell expansion, and IL-6/STAT3 signaling inhibition using Tocilizumab (TCZ) inhibits this expansion [5]. However, the downstream effectors of the IL-6/STAT3 signaling axis that underlie cancer stem cell (CSC) properties are yet unknown.

Conventional cytotoxic cancer treatment is only partly effective because it mainly targets the bulk of cancer cells without affecting the stem-like cancer cells capable of tumor initiation, self-renewal, differentiation, and chemoresistance. The latter highlights the need to circumvent therapy resistance by specifically targeting the stem-like cellular component of malignancies. Thus far, CD44, CD133, and CD166 were shown to represent informative CSC markers in colon cancer [6-9]. Moreover, it has been demonstrated that NANOG, a core homeobox transcription factor (TF) playing key roles in the maintenance and preservation of self-renewal in embryonic stem cells (ESCs), is also expressed in CSCs from different tumor types, including liver and colon cancers [10-13].

FRA1 is a member of the FOS family of TFs encoded by the FOSL1 gene and an important transcriptional downstream target of the IL-6/STAT3 signaling axis leading to CRC aggressiveness through EMT induction [3]. Accordingly, FRA1 is highly expressed in multiple cancers and is thought to play key roles in neoplastic transformation [14], motility [15], cancer drug addiction [16], and stemness [17, 18]. Elevated FOSL1 (FRA1) expression level was reported to result from the activation of the IL-6/STAT3 [3], RASRAF-MEK-ERK-RSK [14, 15], and PKC $\alpha / \theta$ [17, 19] pathways both at the transcriptional and posttranslational levels. In the latter case, phosphorylation of four C-terminal residues, namely, Ser-252, Ser-265, Thr-223, and Thr-230, inhibits FRA1 degradation [15, 19].

Acetylation is a well-known regulatory posttranslational modification. In particular, acetylation at specific residues of several TFs has been shown to represent an important regulatory mechanism. Notably, lysine acetylation is not only restricted to histones but is also found in numerous TFs, including p53, nuclear factor (NF)-кB, and STAT3 [20]. Mechanistically, TF acetylation leads to changes in proteinprotein and protein-DNA interaction [21-23], thus resulting in a plethora of downstream effects including increased/ decreased transcription, protein stabilization, steric prevention of ubiquitination, and chromatin remodeling.

In the present study, we investigated posttranslational regulatory mechanisms downstream of the IL-6/STAT3/
FRA1 inflammatory signaling axis that mediate colon cancer stemness and malignancy and explored novel combinatorial therapeutic approaches to target CRC stem and bulk cells.

\section{Results}

\section{IL-6 promotes colon cancer stemness in an FRA1- dependent manner}

In colon cancer, IL- 6 is known to be secreted by stromal fibroblasts, several types of immune cells, and by parenchymal cancer cells to activate STAT3 signaling, thereby mediating tumor-promoting effects [4]. In view of this, we first excluded autocrine IL- 6 secretion in the two CRC cell lines employed in this study, namely, DLD1 and HT-29, by enzyme-linked immunosorbent assay. As shown in Figure S1a, almost no IL-6 was detected in either cell line, independently of FOSL1 expression. Next, we determined the effects exerted by recombinant IL-6 on both cell lines. Phenotypic analyses by in vitro cell migration, invasion, sphere formation, and chemo-resistance assays and by in vivo lung metastasis assay in nude mice consistently indicated that IL-6 exposure promoted CRC stemness and malignancy (Figure S1b-S1e).

Previously, we reported that IL-6-activated STAT3 upregulates transcription of the FOSL1 gene by directly binding to its promoter and further promoting CRC malignant progression through EMT activation [3]. As shown by western blot analysis in Fig. 1a, IL-6 stimulation resulted in STAT3 pathway activation and up-regulation of FRA1, SOX2, and NANOG expression in both DLD1 and HT-29 cell lines (Fig. 1a). Accordingly, inhibition of the IL-6/ STAT3/FRA1 inflammatory signaling axis by anti-human IL-6R TCZ and by small interfering RNA (siRNA) knockdown of STAT3 significantly reduced sphereformation capacity and chemo-resistance of DLD1 cells upon IL-6 stimulation (Figure S1f and S1g; Fig. 1b).

To determine the effects of IL- 6 on cancer stem cells, we measured by flow cytometry the expression of two colorectal CSCs markers, namely, CD44 and CD133 [6, 7]. Upon IL-6 treatment, the relative proportion of the $\mathrm{CD} 44^{+} / \mathrm{CD} 133^{+} \mathrm{CSC}$ subpopulation increased from $3.3 \%$ to $13.8 \%$ and from $4.4 \%$ to $15.7 \%$ in DLD1 and HT-29, respectively (Figure S1h). Next, we sorted by fluorescenceactivated cell sorter (FACS) the CD $44^{+} / \mathrm{CD} 133^{+}$and CD44 ${ }^{-} / \mathrm{CD} 133^{-}$subpopulations from IL-6-treated DLD1 cells. Sphere-formation and subcutaneous transplantation assays indicated that $\mathrm{CD} 44^{+} / \mathrm{CD} 133^{+}$cells were characterized by increased self-renewal in vitro and tumor-propagating capacity in vivo when compared with $\mathrm{CD} 44^{-} / \mathrm{CD} 133^{-}$cells (Figure S1i and S1j). Of note, IL-6 treatment enhanced these features in both subpopulations. 

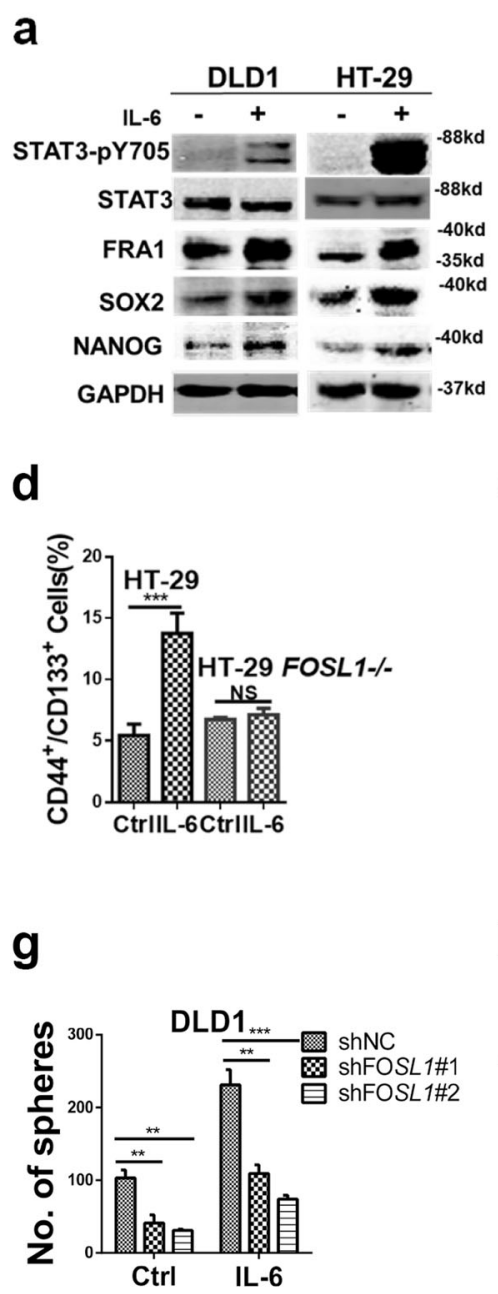

b
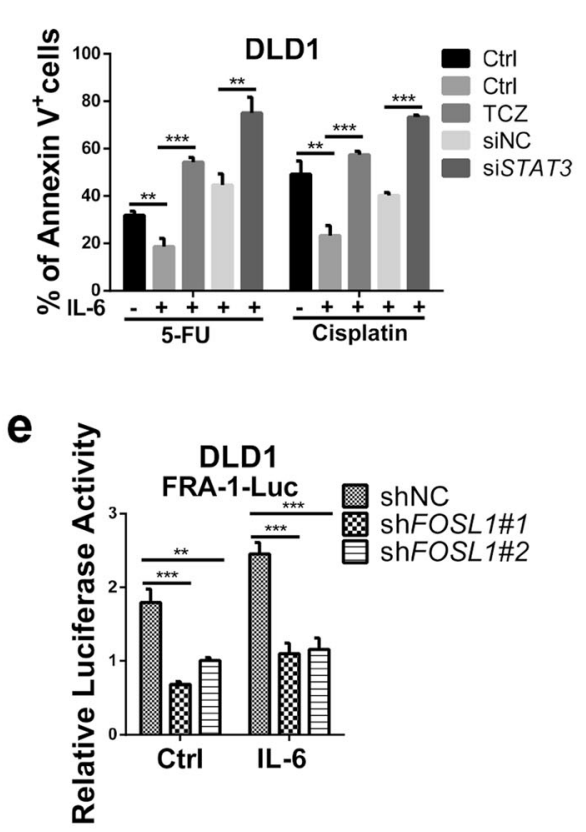

h

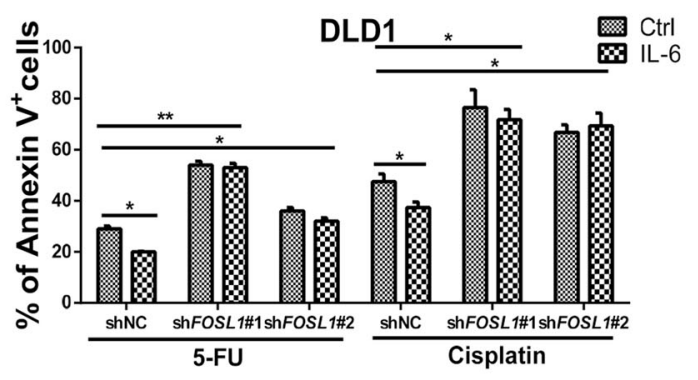

C

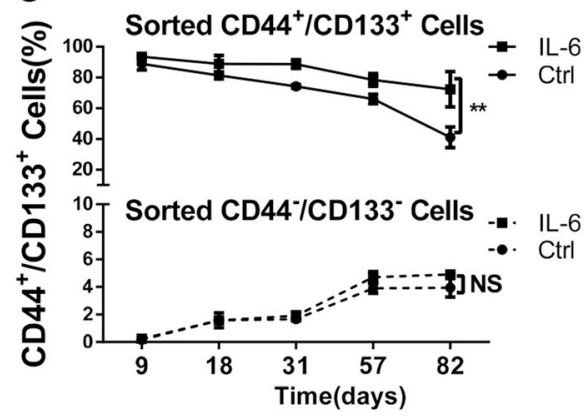

f

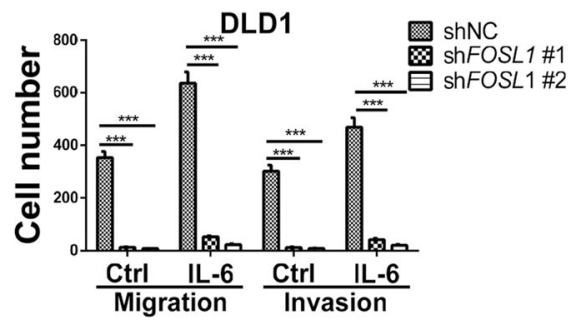

Fig. 1 Interleukin (IL)-6 promotes colon cancer stemness in an FRA1-dependent manner. a Western blot analysis of DLD1 and HT-29 cells cultured in the presence/absence of $50 \mathrm{ng} / \mathrm{ml} \mathrm{IL-6}$ for $24 \mathrm{~h}$. Protein levels of STAT3-pY705, STAT3, FRA1, SOX2, NANOG, and GAPDH were examined. b DLD1 cells were cultured in medium supplemented with the chemotherapeutic drugs 5-Fluorouracil (5-FU) and cisplatin and in the presence/absence of IL-6 $(50 \mathrm{ng} / \mathrm{ml})$, Tocilizumab $(5 \mu \mathrm{g} / \mathrm{ml})$, and siSTAT3. The percentage of apoptotic (Annexin $\mathrm{V}^{+}$) cells were employed as a measure of sensitivity to the treatment when compared to the controls. c CD $44^{+} / \mathrm{CD} 133^{+}$and $\mathrm{CD} 44^{-} / \mathrm{CD} 133^{-}$DLD1 cells were sorted by fluorescence-activated cell sorter (FACS) and cultured in the presence/absence of IL- 6 for the indicated times. These cultures were monitored at the indicated time points by FACS analysis. Quantification of $\mathrm{CD}_{4} 4^{+} / \mathrm{CD} 133^{+}$cells in triplicate (mean $\pm \mathrm{SD}$ ) are depicted. d CD44/CD133 FACS analysis of parental (FOSL1 $\left.{ }^{+/+}\right)$and $F O S L 1^{-I-}$ HT-29 cells cultured in the presence/absence of IL- 6 for 7 days. The relative percentage of the $\mathrm{CD} 44^{+} / \mathrm{CD} 133^{+}$ subpopulation is indicated in the histogram. e FRA1 transcriptional activity was measured in shNC, shFOSL1\#1, and shFOSL1\#2
DLD1 cells cultured in the presence/absence of IL-6 $(50 \mathrm{ng} / \mathrm{ml})$. f, $\mathbf{g}$ Migration and invasion assays (d) and sphere formation assays (e) were performed with non-target control (shNC), shFOSL1\#1, and shFOSL1\#2 DLD1 cells cultured in the presence/absence of IL-6. Results are shown as histograms showing quantitative values of the number of cells (or spheres) from triplicate experiments (mean $\pm \mathrm{SD})$. h shNC and FOSL1 knockdown (shFOSL1\#1 and shFOSL1\#2) DLD1 cells were cultured in medium supplemented with the chemotherapeutic drugs 5-FU and Cisplatin and in the presence/absence of IL-6 $(50 \mathrm{ng} / \mathrm{ml})$. The percentage of apoptotic (Annexin $\mathrm{V}^{+}$) cells indicated that both shFOSL1\#1 and shFOSL1\#2 were characterized by increased sensitivity when compared to the shNC control cells. i $5 \times 10^{5}$ shFOSL1\#2 and, as a control, shNC DLD1 cells were cultured in the presence/absence of IL-6 for 5 days and then injected subcutaneously into the flanks of BALB/C nude mice. DLD1 cells treated with IL-6 increased tumor mass when compared with the shNC group. And this effect was attenuated upon FOSL1 knockdown. * $p<0.05$, $* * p<0.01, * * * p<0.001$. Unpaired $t$ test. Data are presented as mean $\pm \mathrm{SD}$ 
Next, we cultured the sorted cells in the presence/absence of IL-6 and followed their behavior by FACS analysis for 82 days. As depicted in Fig. 1c and Figure S1k, CD44 ${ }^{+} / \mathrm{CD} 133^{+}$DLD1 cells were maintained at a significantly higher percentage under constant IL-6 stimulation, thus confirming its positive effect on the maintenance of the CSC subpopulation.

To assess whether IL-6 affects CSC properties through FRA1, we stably overexpressed the FOSL1 gene in the DLD1 and HT-29 cell lines (Figure S2a). Increased FOSL1 expression enhanced cell features including cell migration and invasion (Figure S2b) and sphere formation (Figure S2c). Accordingly, subcutaneous transplantation of FOSL1overexpressing DLD1 cells into nude mice revealed increased tumorigenicity when compared with the empty vector (EV) group, and IL-6 stimulation enhanced this effect in EV group (Figure S2d).

To further study the role played by FOSL1/FRA1 in IL6-driven colon cancer stemness, FOSL1 gene knockout HT29 cells were constructed by TALEN technology. In FOSL1 ${ }^{-1-}$ HT-29 cells, the IL-6-induced expansion of the CD44 ${ }^{+} / \mathrm{CD} 133^{+}$subpopulation was completely abolished, thus reinforcing the relevance of the IL-6/FRA1 signaling axis in regulating CSCs (Fig. 1d). Moreover, we knocked down FOSL1 (shFOSL1\#1 and shFOSL1\#2) in DLD1 and HT-29 cells using a lentivirus-based approach (Figure S2e). FOSL1 knockdown caused a significant impairment of FRA1 transcriptional activity as measured by the FRA1-Luc reporter activity in the presence/absence of IL- 6 in the culture medium (Fig. 1e). Moreover, the positive in vitro effects of IL-6 on migration, invasion, and sphere formation were dramatically reduced by FRA1 downregulation (Fig. 1f, g; Figure S2f and S2g). In addition, FOSL1 knockdown suppressed the IL-6-induced increase in chemo-resistance to 5-Fluorouracil (5-FU) and Cisplatin in DLD1 and HT-29 cells (Fig. 1h; Figure S2h). Consistent with the above in vitro findings, FOSL1 knockdown also attenuated the tumor-enhancing effect of IL-6 in nude mice (Fig. 1i).

Overall, these results indicated that activation of the IL6/STAT3/FRA1 inflammatory signaling axis promotes CRC stemness and progression toward malignancy.

\section{IL-6/STAT3-driven FRA1 Lys-116 deacetylation increases its transcriptional activity}

Acetylation of non-histone proteins represents a key transcriptional regulatory event [20-23]. In order to elucidate the mechanisms of FRA1 acetylation and its functional consequences, a pan acetyl-lysine antibody (Ace-Lys) was employed. Immunoprecipitation (IP) analysis of ectopically expressed full-length and truncated (Flag- and Myc-tagged) FRA1 in 293T cells showed that the CREB-binding protein (CBP) can acetylate FRA1 and that the acetylated sites were located in the $\mathrm{NH}_{2}$-terminal 130 residues of FRA1 (Figure S3a and S3b). According to an online prediction tool [24], the K116 and K121 residues represent potential FRA1 acetylation sites within the $\mathrm{NH}_{2}$-terminal fragment. Mass spectrometric analysis of DLD1 cells confirmed that both Lys residues were indeed acetylated by CBP (Figure S3c). Of note, these residues were localized within the FRA1 DNA-binding domain (DBD), highly homologous to the corresponding domain in c-FOS. We then generated a structure model of the analogous c-Jun/FRA1 complex bound to the promoter motif showing that the K116 residue was in direct contact with DNA (Fig. 2a). However, alignment of the FRA1 and c-FOS sequences around the K116Ac target region revealed the presence of a key difference, namely the L117 residue in FRA1 corresponding to M149 in c-FOS (Figure S3d). In agreement with these in silico predictions, IP analysis of ectopically expressed FlagEV, Flag-c-Fos and Flag-FRA1 in DLD1 cells showed that K116Ac antibody could hardly recognize c-Fos compared with FRA1 (Figure S3e). As shown in Figure S3f, the DBD of FRA1 and FRA1-K116R reached stability during the last $50 \mathrm{~ns}$ of molecular dynamics (MD) simulations, while the DBD of FRA1-K116Q fluctuated during the entire MD simulation, indicating that the DBDs of FRA1 and FRA1K116R are more stable than that of FRA1-K116Q upon interaction with the target DNA domain. The protein-DNA binding free energies for the three complexes were estimated by MM/GBSA, and the binding affinities for COM, COM-K116Q, and COM-K116R were $-189.97,-187.81$, and $-194.23 \mathrm{kcal} \mathrm{mol}^{-1}$, respectively (Table S1), indicating that the mutation of K116 to Gln weakened the binding of FRA1 to DNA, while the mutation of K116 to Arg strengthened the binding. To clarify the effect of the mutations on the protein-DNA binding quantitatively, the MM/GBSA free energy decomposition was performed. The energy contributions of the residues at position 116 for the DNA binding in the three systems were quite different $\left(\mathrm{K} 116=-5.41 \mathrm{kcal} \mathrm{mol}^{-1}, \quad \mathrm{Q} 116=-4.7 \mathrm{kcal} \mathrm{mol}^{-1}, \quad\right.$ and $\mathrm{R} 116=-6.87 \mathrm{kcal} \mathrm{mol}^{-1}$ ). As for the residue116, the difference was mainly contributed from the polar energy terms $\left(\Delta E_{\text {ele }}+\Delta G_{\mathrm{GB}}=-1.67,-0.61\right.$, and $-2.73 \mathrm{kcal} \mathrm{mol}^{-1}$ for K116, Q116, and R116, respectively) (Figure S3g).

Mutagenesis and luciferase reporter assays provided experimental confirmation of the above results: the FRA1K116R mutant that mimics deacetylation at the Lys-116 residue was characterized by an increment of FRA1 reporter luciferase activity, whereas the acetylation mimic FRA1K116Q/E/A mutants resulted in a reduction of FRA1-luc activity. Notably, the FRA1-K116R/K121R double mutant had similar effects when compared with the single FRA1K116R mutant, while the K121R mutation alone did not affect FRA1 reporter activity. Besides, both the $\Delta 3$ deletion mutant (deletion of 3 C-terminal residues) and the 

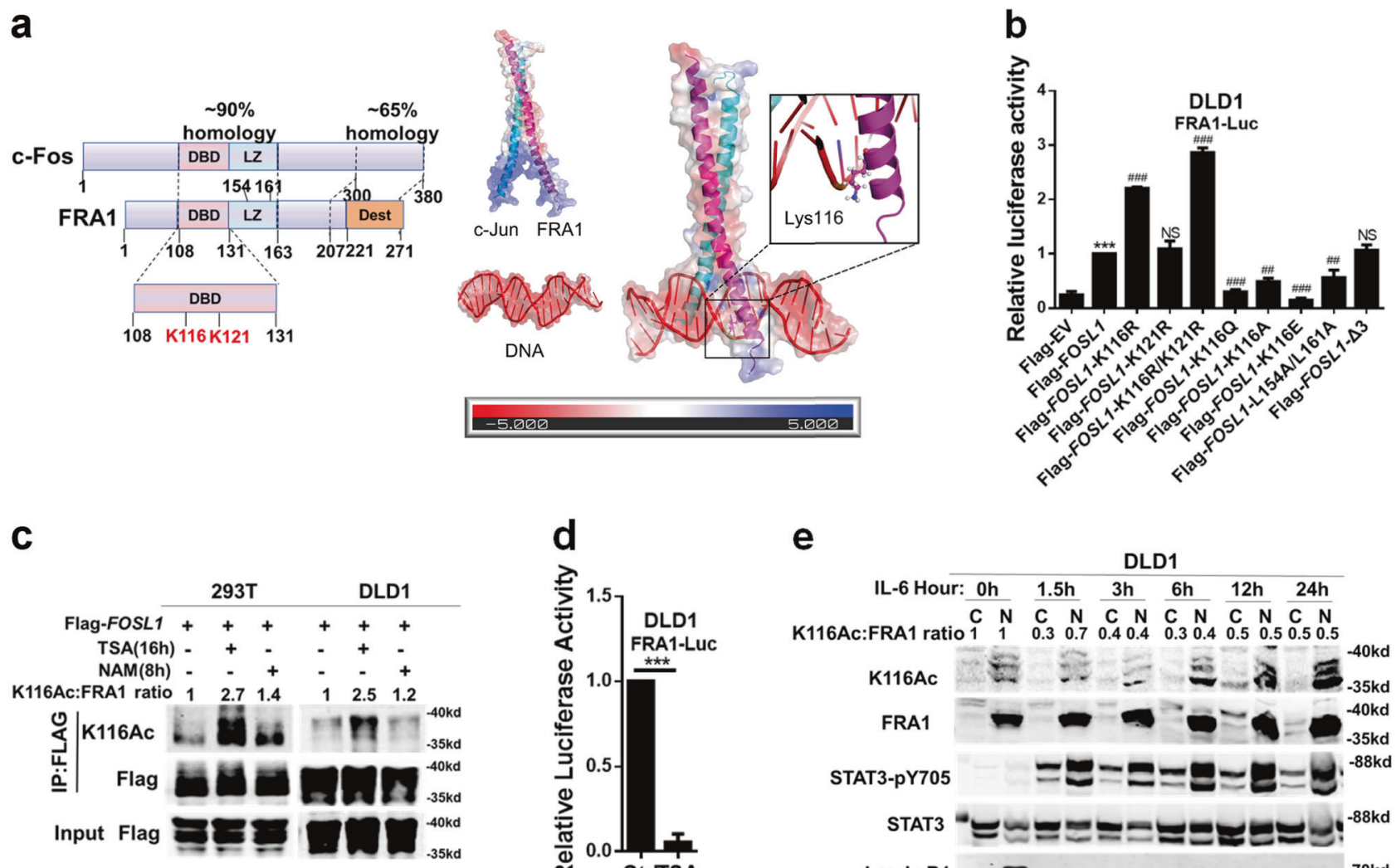

f

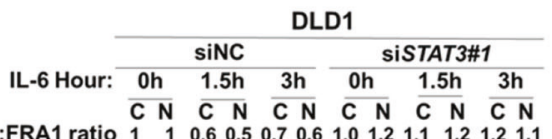

K116Ac:FRA1 ratio $\begin{array}{lllllllllllll}1 & 1 & 0.6 & 0.5 & 0.7 & 0.6 & 1.0 & 1.2 & 1.1 & 1.2 & 1.2 & 1.1\end{array}$

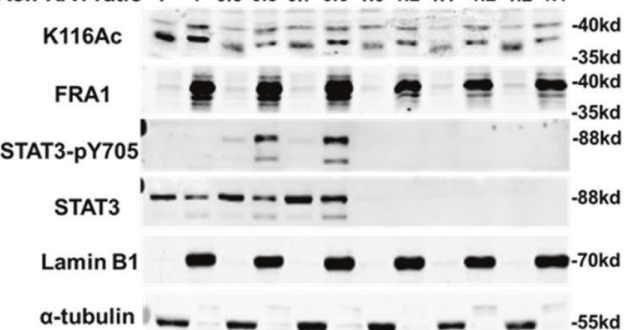

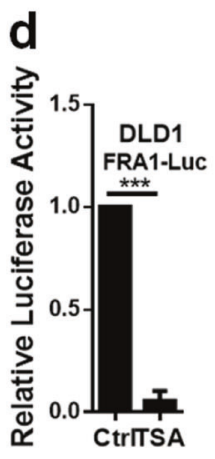

e

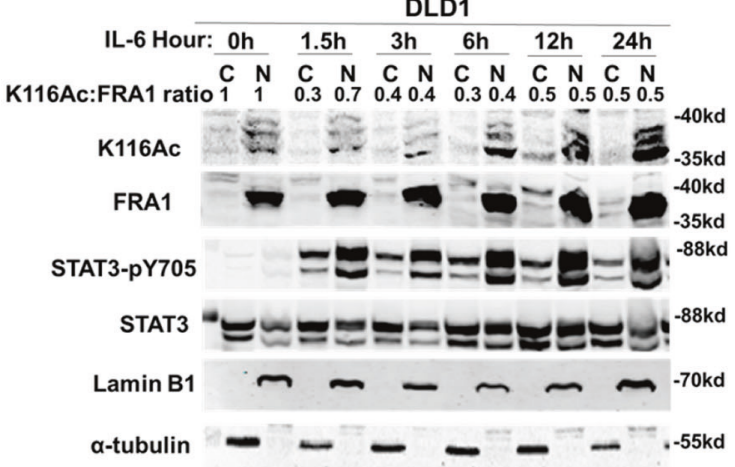

dimerization-defective (L154A/L161A) mutant of FRA1 [25] did not affect FRA1-luc activity (Fig. 2b). Furthermore, additional experimental evidence excluded that the K116 and K121 residues of FRA1 were involved in protein stability and in its interaction with c-Jun (Figure S4a and S4b).

To enable the analysis of K116 deacetylation in endogenous FRA1, we generated a polyclonal antibody specifically recognizing the acetylated Lys-116 residue. The specificity of the anti-K116Ac antibody was validated by western blot and IP analyses (Figure S4c and S4d). Additional western blot analysis with the K116Ac antibody showed an increase of approximately 2-3-fold in K116 acetylation by trichostatin A (TSA; an inhibitor of histone deacetylase HDAC family I, II, and IV) but not by nicotinamide (NAM; an inhibitor of the SIRT family deacetylase), in both $293 \mathrm{~T}$ and DLD1 cells (Fig. 2c). TSA treatment did reduce FRA1 luciferase activity (Fig. 2d). Hence, these results indicate that K116 deacetylation plays a key role in the regulation of FRA1 transcriptional activity.

Since IL-6/STAT3 signaling positively affects FOSL1 transcription and thereby increases CRC stemness as shown above, we asked whether FRA1 K116 deacetylation could be directly involved in the modulation of CSCs properties. As shown in Fig. 2e, FRA1 mainly localizes to the nucleus of CRC cells in agreement with its function as a TF. After a 
Fig. 2 Interleukin (IL)-6/signal transducer and activator of transcription 3 (STAT3)-driven FRA1 Lys-116 deacetylation increases its transcriptional activity. a Left panel: schematic diagram of the homology between structural domains in c-Fos and FRA1. The highest homology is found in the DNA-binding domain that encompasses the two FRA1 acetylated lysine residues (K116 and K121) identified by mass spectrometry. Two leucine residues (L154 and L161) located in the Leucine zipper domain (LZ) were responsible for FRA1 heterodimerizing with the JUN family. Phosphorylation of many serine (S) or threonine (T) residues of FRA1 in destabilizer domain (DEST) increase its stability. Right panel: Charge density map of AP-1(c-Jun and FRA1), DNA and the complex. Lys116 in FRA1 chain is shown as sticks and spheres. b Relative luciferase activity in DLD1 cells cotransfected with wild-type and (deletion and point) mutant FOSL1 constructs, together with pRL-TK as an internal control reporter. $\Delta 3$ deletion and L154A/L161A mutant constructs were here employed as controls $(* p<0.05, * * p<0.01, * * * p<0.001$, compared with the Flag$\mathrm{EV} ;{ }^{\#} p<0.01,{ }^{\# \#} p<0.001$, compared with the Flag-FOSL1). c Immunoprecipitation (IP) analysis of FRA1 K116 acetylation. The Flag-FOSL1 construct was transfected into 293T and DLD1 cells, followed by treatment with the deacetylase inhibitors trichostatin A (TSA) or nicotinamide, and IP with antibodies directed against Flag. FRA1 acetylation was analyzed by western blot with the K116Acspecific antibody. d FRA1 transcriptional activity in response to TSA treatment for $16 \mathrm{~h}$ and then measured by FRA1-Luc luciferase assay in DLD1 cells. e DLD1 cells were cultured in the presence of IL-6 for the indicated times and the cytoplasmic and nuclear protein fractions were separated for western blot analysis with the indicated antibodies. K116 acetylation was detected with the K116Ac antibody and normalized against the FRA1 protein. (LaminB1 and $\alpha$-tubulin were employed as nuclear and cytoplasmic protein control, respectively). f DLD1 cells were transfected with siNC or STAT3 small interfering RNAs and incubated with IL-6 for the indicated times. Cytoplasmic and nuclear protein fractions were separated for western blot analysis using the indicated antibodies. K116 acetylation was detected with K116Ac antibody and normalized against the FRA1 protein. g, h HT-29 FOSL1 $^{-l-}$ cells were constructed by TALEN and transfected with expression constructs encompassing wild-type and mutated FOSL1. These cells were then analyzed for their capacity to form spheres in vitro (g) and to form subcutaneous tumors in vivo upon IL-6 stimulation (h). $* p<0.05, * * p<0.01, * * * p<0.001$. Unpaired $t$ test. Data are presented as mean \pm SD

short ( $0-3 \mathrm{~h})$ exposure to IL-6, K116 acetylation level readily decreased while the overall expression level of FRA1 remained unchanged. Prolonged IL-6 treatment (6$24 \mathrm{~h}$ ) further increased FOSL1 transcription, though with a decrease in the relative level of K116-acetylated FRA1 versus total FRA1 in both nucleus and cytoplasm (Fig. 2e). Accordingly, siRNA-mediated STAT3 knockdown markedly attenuated the IL-6-induced FOSL1 expression and K116 deacetylation (Fig. 2f). Moreover, in vitro and in vivo assays showed that cells expressing wild-type FRA1 or the deacetylation mimic K116R were characterized by a significant increase in sphere formation and tumorigenic potential when compared with the K116Q acetylation mimic construct (Fig. 2g, h).

Taken together, these data showed that activation of the IL-6/STAT3 signaling axis increases FRA1 level not only by its transcriptional upregulation but also by posttranslational deacetylation at its $\mathrm{K} 116$ site, thus promoting colorectal CSC properties.

\section{HDAC6 deacetylates FRA1 and underlies its transcriptional activation downstream of IL-6/STAT3}

Treatment with the HDAC inhibitor TSA, though not with the SIRT inhibitor NAM, increased K116 acetylation (Fig. 2c), thus indicating that HDACs may underlie FRA1 deacetylation. To provide experimental evidence for this, we first analyzed endogenous HDAC1-6 levels in the colon cancer cell lines DLD1, HT29, and 293T and showed that all six deacetylases are expressed albeit at different levels (Figure S5a). Next, we expressed HDAC1-6 individually in DLD1 cells. As shown in Fig. 3a, only HDAC6, an HDAC family II deacetylase that mainly targets non-histone proteins, decreased the K116 acetylation level of endogenous FRA1 in CRC cells. Moreover, treatment with Romidepsin (FK228; HDAC1/2 inhibitor) or Entinostat (MS-275; HDAC1/3 inhibitor) had no evident impact on K116 acetylation (Figure S5b). Accordingly, treatment with the specific HDAC6 inhibitor Tubastatin A increased K116 acetylation and decreased FOSL1 luciferase activity (Fig. 3b). Similar results were obtained by the HDAC6 knockdown in DLD1 cells (Fig. 3c). In agreement with these results, western blot analysis revealed that HDAC6 is localized in both the nucleus and cytoplasm of CRC cells (Figure S5c).

We then examined the physical interaction between HDAC6 and FRA1 by co-IP analysis. Both ectopic and endogenous HDAC6 could be coprecipitated by FRA1 in 293 T and DLD1 cells (Fig. 3d, e). Moreover, this interaction was enhanced by IL-6 and attenuated by siSTAT3 (Fig. $3 \mathrm{e}$ ), thus indicating that IL-6/STAT3 signaling may positively affect the interaction of HDAC6 with FRA1 and facilitate its deacetylation. Last, siRNA-mediated STAT3 knockdown markedly attenuated IL-6-induced K116 deacetylation but had no effect on global HDAC6 expression (Fig. 3f).

Overall, these results indicated that HDAC6 underlies K116 deacetylation of FRA1 and its transcriptional activation downstream of IL-6/STAT3.

\section{FRA1 trans-activates NANOG expression upon K116 deacetylation}

To further explore direct transcriptional targets of FRA1 in the regulation of colon cancer stemness, we analyzed NANOG protein expression in FOSL1-overexpressing DLD1 and HT-29 cells by western blot (Fig. 4a; Figure S6a). FOSL1 expression resulted in an increment of NANOG in both cell lines. Moreover, overexpression of FOSL1 enhanced the transcriptional activities of the 
a

DLD1

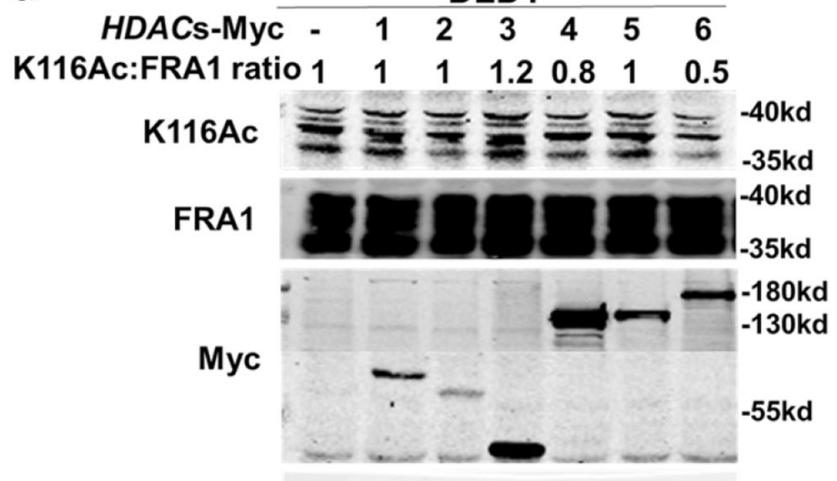

a-tubulin I

C
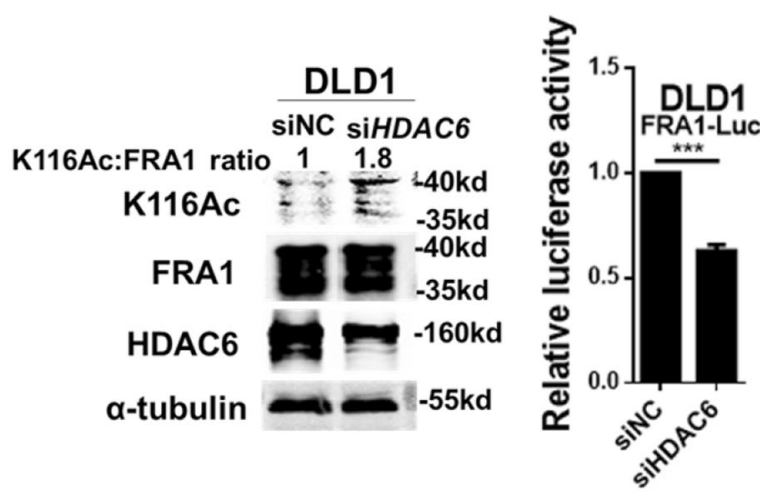

e

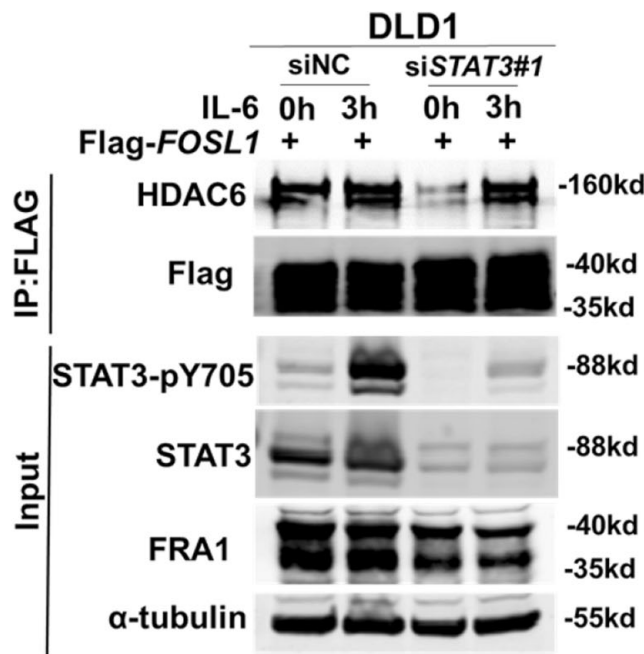

Fig. 3 HDAC6 deacetylates FRA1 and underlies its transcriptional activation downstream of interleukin (IL)-6/signal transducer and activator of transcription 3 (STAT3). a Overexpression of HDAC6, but not of other $H D A C$ s, decreases FRA1 acetylation levels. Each of the Myc-tagged HDAC (1-6) were transfected into DLD1 cells and the K116 FRA1 acetylation levels were determined by western blotting with the K116Ac antibody and normalized against the FRA1 protein. b Western blot analysis and relative FRA1-luc reporter activities in DLD1 cells treated with the HDAC6 small inhibitor Tubastatin A for the indicated times. c Small interfering RNA (siRNA) oligonucleotides directed against HDAC6 were employed to transfect DLD1 cells. The levels of endogenous K116 acetylation, FRA1, and HDAC6 b
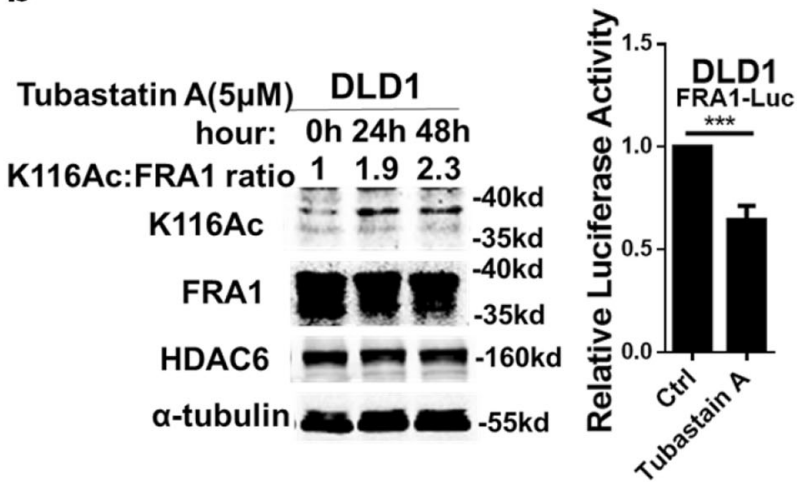

d

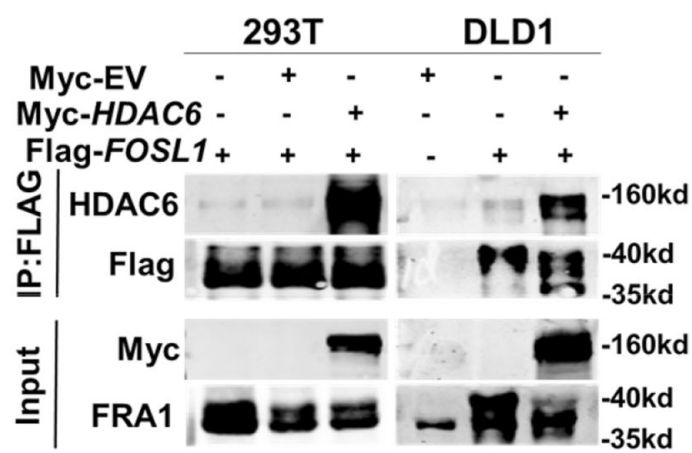

f

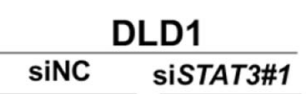

IL-6 Oh 1.5h 3h 0h 1.5h 3h

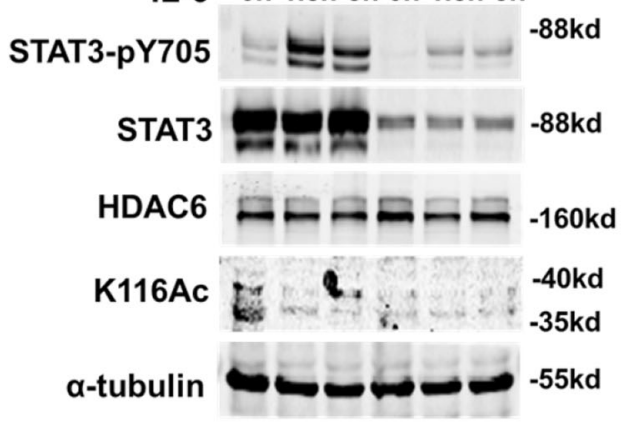

expression were analyzed by western blot. And the FRA1-luc reporter activity was assayed. d IP-Flag analysis of the interaction between FRA1 and HDAC6 in 293T and DLD1 cells transfected with the indicated plasmids. e DLD1 cells were transfected with Flag-FOSL1 and the indicated siRNAs, followed by IL- 6 stimulation for $3 \mathrm{~h}$. IPFlag analysis of HDAC6 was performed to assess the role of the IL-6/ STAT3 signaling pathway on the FRA1-HDAC6 interaction. f DLD1 cells were transfected with non-target control oligonucleotides (siNC) or STAT3 siRNAs and incubated with IL-6 for the indicated times. The levels of STAT3-pY705, STAT3, HDAC6, $\alpha$-tubulin, and endogenous K116 acetylation protein were determined by western blot. $* * * p<0.001$. Unpaired $t$ test. Data are presented as mean $\pm \mathrm{SD}$ 


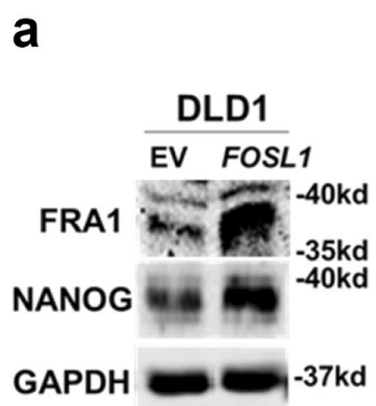

b

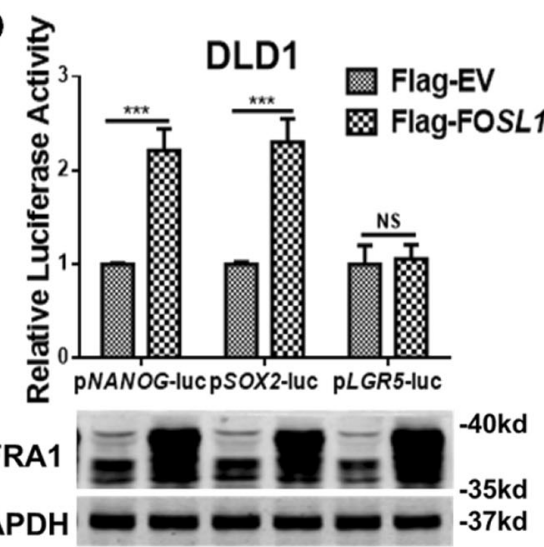

C

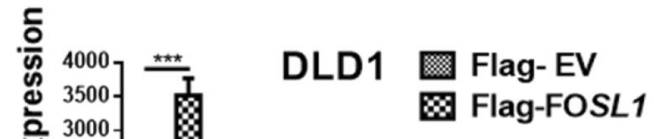

d

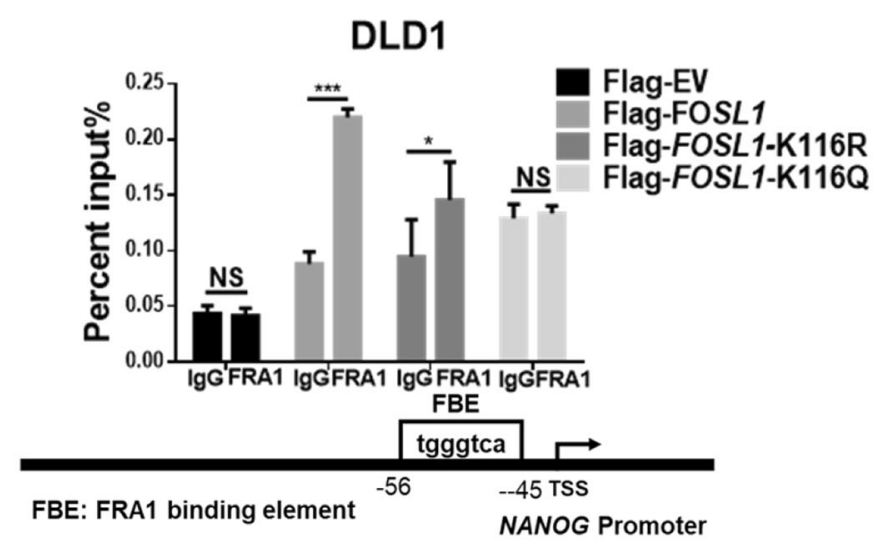

$\mathbf{f}$

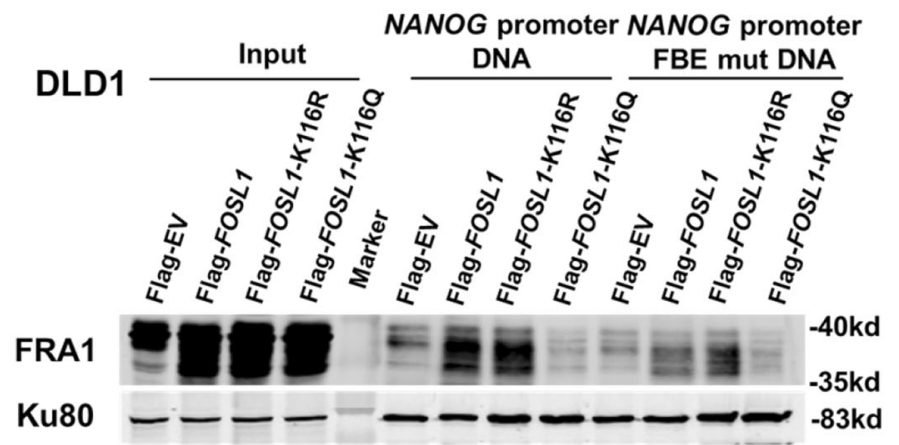

世 $2000-8$

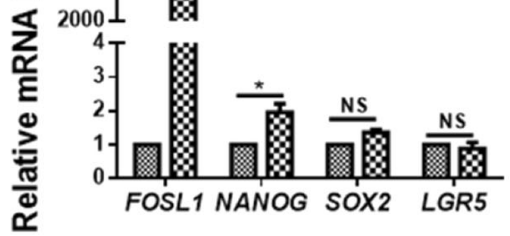

$\stackrel{\mathbf{\alpha}}{\mathbf{\alpha}}$

e

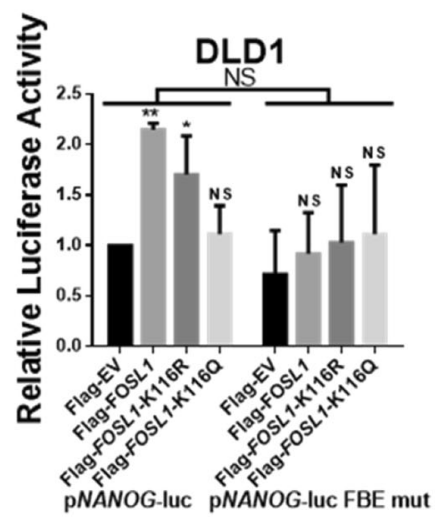

g

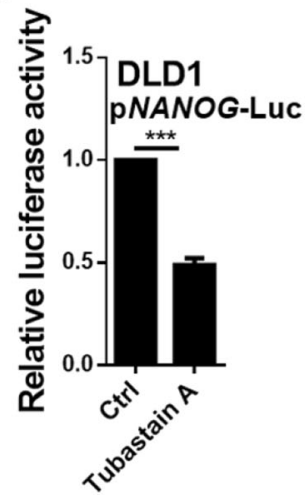

Fig. 4 FRA1 trans-activates $N A N O G$ expression upon K116 deacetylation. a FRA1 and NANOG expression analysis by western blot in empty vector (EV) and FOSL1-overexpressing DLD1 cells. b, c Relative reporter luciferase activity (b) and mRNA levels (c) of NANOG, SOX2, and LGR5 in EV and FOSL1-overexpressing DLD1 cells. d Chromatin immunoprecipitation (ChIP) analysis of DLD1 cells transfected with Flag-tagged wild-type and mutated FOSL1 expression vectors. ChIP was performed with the Flag antibody. Quantitative reverse transcriptase-polymerase chain reaction analysis was performed on immune-precipitated DNAs using a primer

NANOG-luc and SOX2-luc reporters in DLD1 cells but not of LGR5-luc (Fig. 4b). Quantitative reverse transcriptasepolymerase chain reaction (PCR) analysis confirmed the pair specific for the $N A N O G$ promoter. e Relative luciferase activity of wild-type and FBE-mutant (predicted FRA1-binding site) NANOG-luc reporters in DLD1 cells transfected with wild-type and mutant FOSL1 expression constructs. f DNA pull-down assay analysis of FRA1binding ability to the biotin-labeled wild-type and FBE-mutant $N A N O G$ promoter probe $(-404 /-1)$ in DLD1 cells stably overexpressing wild-type and mutant FOSL1. Ku80 served as a control. $\mathrm{g}$ Relative luciferase activity of the $N A N O G$-Luc reporter in DLD1 cells upon Tubastatin A treatment for $8 \mathrm{~h}$. * $p<0.05, * * p<0.01$, ***p $p<$ 0.001 . Unpaired $t$ test. Data are presented as mean $\pm \mathrm{SD}$

upregulation of endogenous $N A N O G$ gene expression, though not of SOX2 and LGR5, in FOSL1-overexpressing DLD1 cells (Fig. 4c). 
Clinical validation of these results was provided by the positive correlation between FOSL1 (encoding for FRA1) and NANOG mRNA level in one CRC patient cohort from the GSE24551-GPL5175 [26] dataset (Figure S6b).

To evaluate whether $N A N O G$ is directly regulated by FRA1, we first analyzed in silico its promoter sequence using the JASPAR database (http://jaspar.genereg.net/) and predicted a FRA1/AP-1-binding site (FBE) 45-56 nucleotides upstream of the translational start site. Chromatin IPquantitativePCR analysis confirmed that in DLD1 cells both wild-type FRA1 and FRA1-K116R, though not FRA1$\mathrm{K} 116 \mathrm{Q}$, bind to the NANOG gene promoter through the FBE site (Fig. 4d). Similar results were obtained by $N A N O G$ gene luciferase reporter assays and by $N A N O G$ gene promoter DNA pull-down assays, respectively (Fig. 4e, f). Moreover, treatment with the HDAC6 inhibitor Tubastatin A significantly decreased NANOG-luc activity (Fig. 4g).

These results indicate that FRA1 upregulates $N A N O G$ gene transcription by directly interacting with its promoter. FRA1 deacetylation at K116 promotes its binding to the $N A N O G$ promoter.

\section{NANOG is a key downstream effector of IL-6/STAT3/ FRA1-driven CSC properties}

In view of the IL-6/STAT3-driven enhanced expression of both FOSL1 and NANOG (Figs. 1a and 5a), and of the effects of FRA1 on $N A N O G$ transcriptional activity, we further investigated the role played by key $\mathrm{TF}$ as the downstream effector of the IL-6/STAT3/FRA1 signaling axis in CSC properties. We showed that siRNA-mediated STAT3 knockdown markedly attenuated IL-6-induced FRA1 and NANOG expression levels in DLD1 cells (Fig. 5a). Notably, FOSL1 gene knockout significantly blocked IL-6-induced NANOG expression without affecting STAT3 activation in HT-29 cells (Fig. 5b). Thus NANOG is a downstream effector of FRA1 in response to IL-6/ STAT3 stimulation.

Next, we overexpressed NANOG in DLD1 cells with reduced FOSL1 expression (FOSL1 knockdown) (Figure S6c). On its own, FOSL1 knockdown greatly reduced chemoresistance to 5-FU and Cisplatin in DLD1 cells, and this inhibitory effect was accordingly rescued upon NANOG overexpression (Fig. 5c). Similar results pointing to an increment of CSCs upon NANOG expression were obtained by sphere-formation and mouse xenograft assays (Fig. 5d, e).

We then knocked down NANOG in DLD1 and HT-29 cells using a lentivirus-based shRNA approach (Figure S6d). The positive effects of IL-6 on migration, invasion, sphere-formation ability, and chemoresistance were significantly reduced by $N A N O G$ knockdown (Fig. 5f-h; Figure S6e-S6g). Consistent with the in vitro findings, $N A N O G$ knockdown abolished the tumor-enhancing effect of IL-6 (Fig. 5i). These data pointed at $N A N O G$ as an important downstream effector of the IL-6/STAT3/ FRA1 signaling axis in the modulation of CSC properties.

\section{Increased FRA1 protein expression with low K116 acetylation correlate with IL-6 and NANOG levels and with poor prognosis among CRC patients}

To validate the correlation between malignant behavior of CRC and the expression levels of IL-6, K116Ac-FRA1, FRA1, and NANOG, a cohort of prospectively collected CRCs $(n=123)$ was employed. Immunofluorescence analysis showed increased expression and nuclear colocalization of FRA1, phosphorylated STAT3, and NANOG protein in the cancer samples when compared with adjacent (para-carcinoma) normal tissues (Fig. 6a; Figure S6h). Immunohistochemical (IHC) analysis with the antiK116Ac antibody (whose specificity was previously validated; Figure S7a) showed that FRA1 was mostly identified in the nucleus of cancer cells while its K116-acetylated form mainly localized to the cytoplasm (Fig. 6b; Figure S7b), thus indicating that phosphorylated STAT3 can activate FRA1 both by transcriptional upregulation and deacetylation leading to increased NANOG expression levels.

Using consecutive tissue sections, IHC analysis further confirmed the positive correlation between the expression level of FRA1 and IL-6 secretion (Fig. 6c). Notably, cancer cells located in marginal areas adjacent to inflammatory regions displayed stronger FRA1 immunoreactivity as well as weaker K116Ac immunoreactivity when compared with those in the center of the cancer mass. The latter observations suggest a paracrine effect of inflammatory cells on FRA1 protein expression and K116 deacetylation on CRC cells. HDAC6 localizes to both the cytoplasm and the nucleus of cancer cells (Fig. 6c). Moreover, quantitative analysis of the IHC results indicated that HDAC6 levels inversely correlated with FRA1 acetylation at K116 in the CRC cohort (Fig. 6d). IL-6 and FRA1 protein expression was increased in tumor compared with non-tumor tissues and positively correlated in the CRC cohort (Figure S7c$\mathrm{S} 7 \mathrm{e})$. These results were further validated with the GSE24551-GPL5175 dataset (Fig. 6e; Figure S7f and S7g).

Last, we evaluated the prognostic value of increased FRA1 combined with its decreased K116 acetylation in the prospective CRC cohort. CRC patients with high FRA1 and relatively low K116Ac expression are characterized by a significantly reduced overall survival when compared with those with carcinomas characterized by low FRA1 and relatively high K116Ac expression (Fig. 6f, g). 


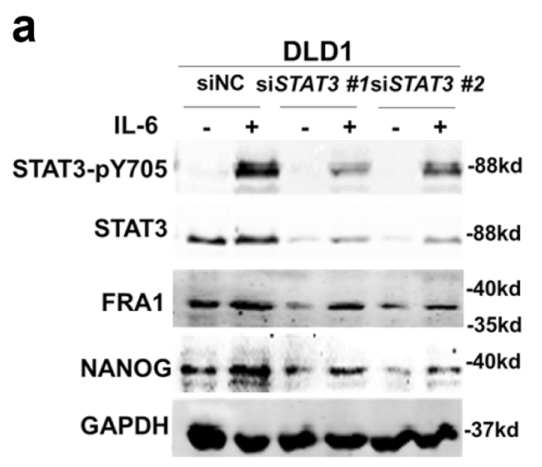

d

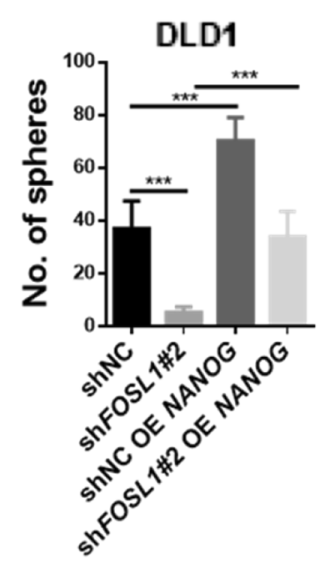

g

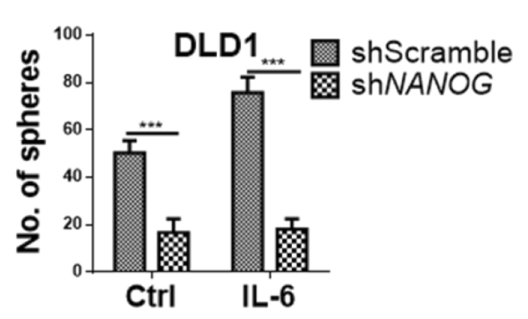

b

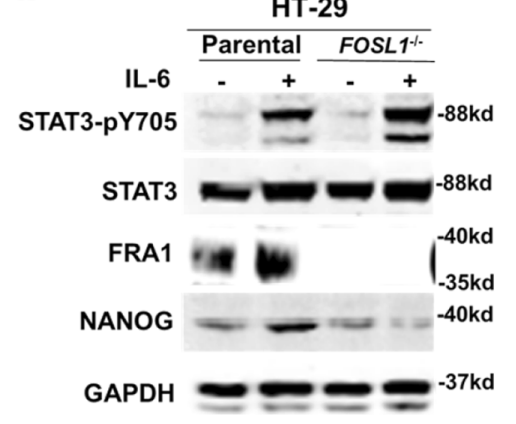

e

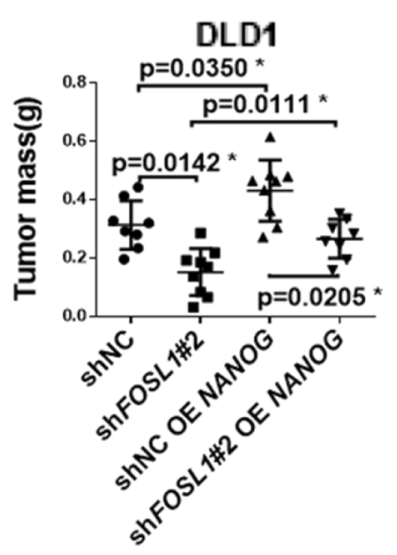

h

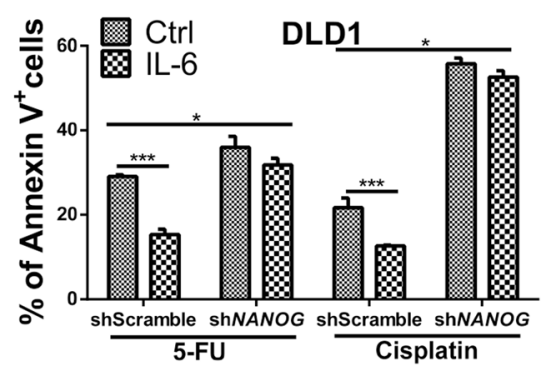

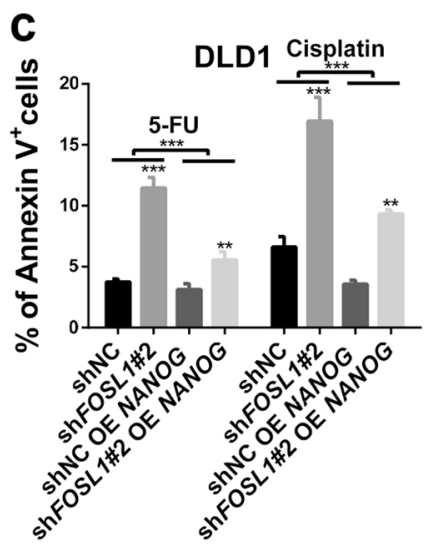

f

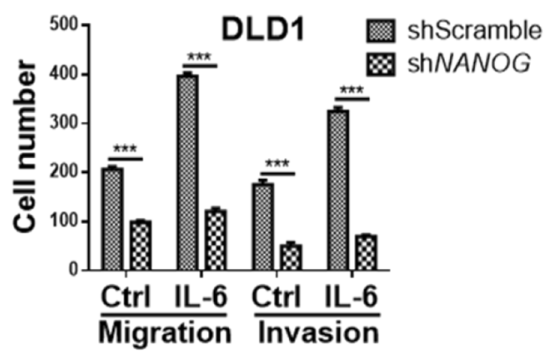

i

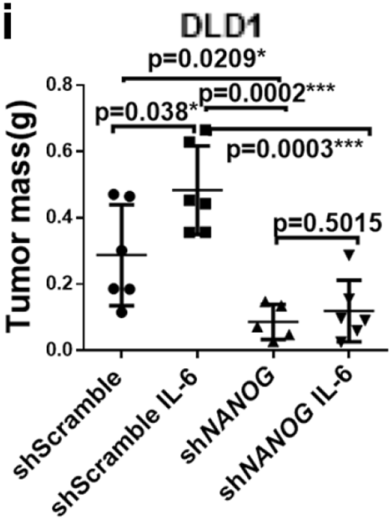

\section{Combined treatment of 5-FU with the HDAC6 inhibitor Tubastatin A synergistically inhibits CRC stem-like properties and malignant growth}

Based on the results thus far, inhibition of the enzymatic machinery leading to FRA1-K116 deacetylation is expected to result in improved clinical outcomes especially in patients with increased IL-6 and FRA1 protein expression. To provide proof of principle for this concept, we first sorted and cultured $\mathrm{CD} 44^{-} / \mathrm{CD} 133^{-}$and $\mathrm{CD} 44^{+} / \mathrm{CD} 133^{+}$ DLD1 cells in the presence/absence of IL- 6 for 18 days to then harvest them and separate the cytoplasmic and nuclear protein fractions for western blot analysis. $\mathrm{CD} 44^{+} / \mathrm{CD} 133^{+}$ CSCs are characterized by lower K116 acetylation levels both in the nucleus and cytoplasm compared with their double-negative $\left(\mathrm{CD} 44^{-} / \mathrm{CD} 133^{-}\right)$counterparts (Fig. 7a). Upon IL-6 stimulation, FRA1-K116 deacetylation was observed in $\mathrm{CD} 44^{+} / \mathrm{CD} 133^{+}$but not in $\mathrm{CD} 44^{-} / \mathrm{CD} 133^{-}$ cells. Likewise, we detected decreased K116Ac levels in $\mathrm{CD}_{4} 4^{+} / \mathrm{CD} 133^{+}$compared with $\mathrm{CD} 44^{-} / \mathrm{CD} 133^{-}$ DLD1 cells (Fig. 7b). These results indicate that colorectal CSCs are relatively low in acetylated FRA1-K116 and that IL-6 can further lower the relative level of the modified protein in these stem-like cancer cells. 
Fig. $5 N A N O G$ is a key downstream effector of interleukin (IL)-6/ signal transducer and activator of transcription 3 (STAT3)/FRA1-driven CSC properties. a DLD1 cells were transfected with non-target control (siNC) or STAT3 small interfering RNAs (siRNAs) and incubated with IL-6 for the indicated times. The protein levels of STAT3pY705, STAT3, FRA1, NANOG, and GAPDH was examined by western blot analysis with the respective specific antibodies. b Parental $\left(\right.$ FOSL $\left.^{+/+}\right)$and FOSL1 $1^{-1-}$ HT-29 cells were cultured in the presence/ absence of IL-6 for $24 \mathrm{~h}$. The protein levels of STAT3-pY705, STAT3, FRA1, NANOG, and GAPDH was examined by western blot analysis with the respective specific antibodies. c-e Chemo-resistance (c), sphere (d), and xenograft (e) formation assays of DLD1 cells featuring different combinations of FOSL1 knockdown, NANOG overexpression, and IL- 6 stimulation $(\mathrm{shNC}=$ control for the siRNAdriven FOSL1 knockdown). f, g Migration and invasion (f) and sphere formation (g) assays were performed with shScramble (control for the shRNA-driven $N A N O G$ knockdown) and shNANOG DLD1 cells cultured in the presence/absence of IL-6. Results are shown as histograms showing quantitative values of the number of cells (or spheres) from triplicate experiments (mean $\pm \mathrm{SD}$ ). h shScramble and $\operatorname{shNANOG}$ DLD1 cells were cultured in medium supplemented with the chemotherapeutic drugs 5-Fluorouracil and Cisplatin and in the presence/ absence of IL-6 $(50 \mathrm{ng} / \mathrm{ml})$. The percentage of apoptotic $\left(\right.$ Annexin $\left.\mathrm{V}^{+}\right)$ cells indicate that $\operatorname{sh} N A N O G$ DLD1 cells were characterized by increased sensitivity when compared to the shScramble cells. IL-6 enhances chemo-resistance in shNC DLD1 cells, an effect that is abrogated by the NANOG knockdown. i $5 \times 10^{5}$ shScramble and shNANOG DLD1 cells were cultured in the presence/absence of IL-6 for 5 days and then injected subcutaneously into the flanks of BALB/C nude mice. In the shScramble group, DLD1 cells treated with IL-6 formed larger tumors, an effect that was abolished upon NANOG knockdown. $* p<0.05, * * p<0.01, * * * p<0.001$. Unpaired $t$ test. Data are presented as mean $\pm \mathrm{SD}$

To investigate whether the combination of 5-FU and Tubastatin $\mathrm{A}$ is effective in targeting colon cancer stem cells and tumor growth, in vitro drug resistance and in vivo mouse xenograft assays were performed using sorted $\mathrm{CD} 44^{+} / \mathrm{CD} 133^{+}$and $\mathrm{CD} 44^{-} / \mathrm{CD} 133^{-}$DLD1 cells. We showed that, while $\mathrm{CD} 44^{+} / \mathrm{CD} 133^{+}$cells were more resistant to 5-FU or Tubastatin A employed as single agents when compared with $\mathrm{CD} 44^{-} / \mathrm{CD} 133^{-}$(Fig. 7c; Figure S8a), the combination of 5-FU with the HDAC6 inhibitor reversed their drug resistance phenotype (Fig. 7d; Figure $\mathrm{S} 8 \mathrm{~b}$ ). Western blot analysis of the cells treated with the single and combined agents as in Fig. $7 \mathrm{~d}$ showed that Tubastatin A alone or combined with 5-FU treatment resulted in increased expression of K116Ac and decreased expression of NANOG (Figure S8c). The 5-FU/Tubastatin A combination also had synergistic inhibitory effects on DLD1 cell proliferation (Figure S8d). Accordingly, the combined 5-FU/Tubastatin A treatment dramatically inhibited xenograft growth in vivo (Fig. 7e). Of note, the toxicity of the combined treatment was not higher than that of the single agents, as shown by the unchanged body weight among the different treated animals (Figure S8e). The results are also supported by the observed positive correlation between HDAC6 and NANOG expression in the GSE24551-GPL5175 dataset (Figure S8f).
Overall, these results indicate that the combined treatment of the chemotherapeutic agent 5-FU with the HDAC6 inhibitor Tubastatin A may prove to be a potential novel curative approach for CRC patients, especially in those cases where the IL-6/STAT3/FRA1 signaling axis is activated.

\section{Discussion}

In this study, we show that FRA1 is a key downstream effector of the IL-6-STAT3 signaling axis and that it accounts for increased colon cancer stemness and malignant behavior in the context of inflammation. We find that, upon IL-6/STAT3 signaling, HDAC6-driven deacetylation of a previously unrecognized K116 residue in FRA1 activates its transcriptional activity. Among the direct transcriptional targets of FRA1, NANOG plays a central role in modulating CRC stemness. Accordingly, increased FRA1 expression combined with low levels of K116 nuclear acetylation correlate with IL-6 secretion and with poor prognosis and overall survival among CRC patients. Notably, the combination of 5-FU with the HDAC6 inhibitor Tubastatin A may offer a potential novel therapeutic approach for colorectal CSCs.

Increasing evidence has shown that, upon inflammation, IL-6 activates STAT3 and exerts its predominant function in the regulation and maintenance of liver and colon CSCs [5, 27] However, the underlying molecular mechanisms and the IL-6/STAT3 downstream target genes that account for increased cancer stemness and malignancy are to date yet largely obscure. Previously, we reported that aberrant activation of STAT3, but not AKT or extracellular signalregulated kinase (ERK), mediated the IL-6-induced upregulation of FRA1, required for EMT activation and increased CRC aggressiveness [3]. Here we employed human recombinant IL-6 to activate the IL-6/STAT3 pathway and found that it promotes colon cancer stemness in an FRA1-dependent manner. Consistent with our findings, the role of FRA1 in regulating CSC properties has also been reported in breast and liver cancer [17, 18]. In addition to IL-6, another inflammatory cytokine, IL-22, was also reported to promote pancreatic and CRC stemness via STAT3 activation [28, 29], which suggested that these two cytokines might coordinate with each other to modulate colorectal CSC properties.

The activation of FRA1 downstream of IL-6/STAT3 is known to mainly occur at the transcriptional level through pSTAT3-dependent upregulation of the FOSL1 gene. However, more recent studies have revealed that protein modifications, e.g., phosphorylation of S265, T223, and T230 [17, 19], are critical for FRA1 stabilization and for the modulation of its downstream target genes. Also, FRA1 


\section{a}

$60 \mathrm{X}$ DAPI
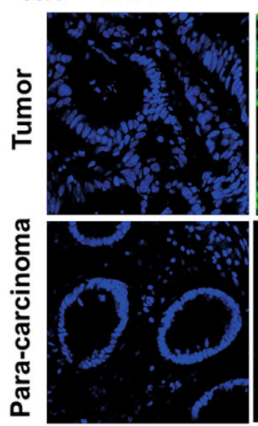

C

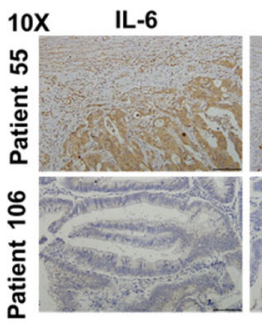

FRA1
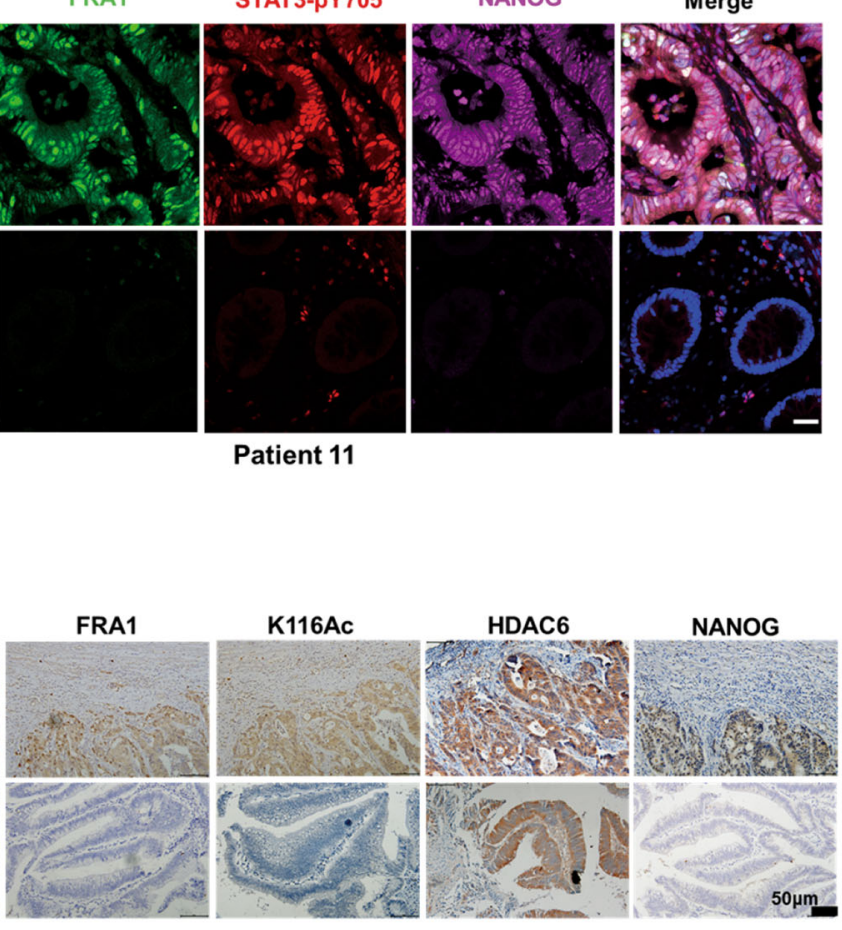

Patient 11
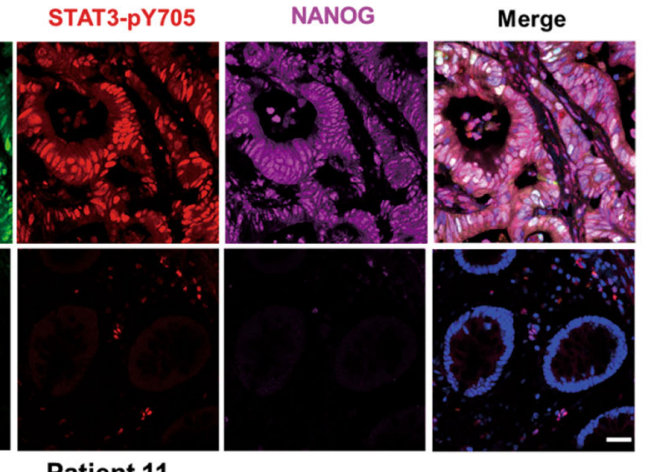

e

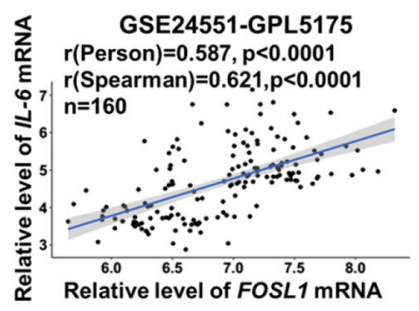

$f$

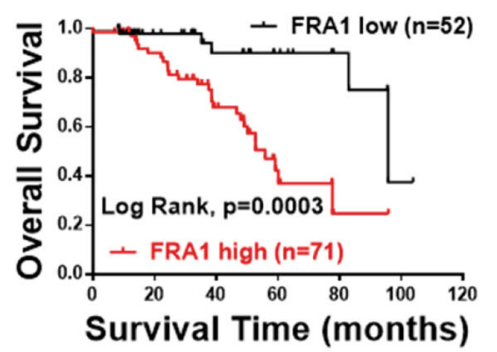

b

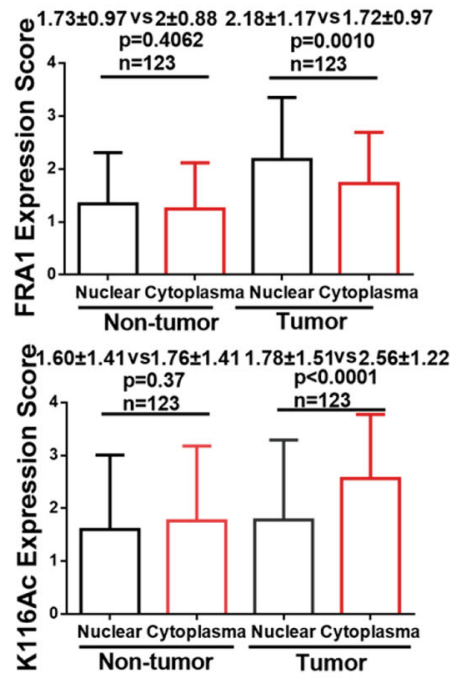

d

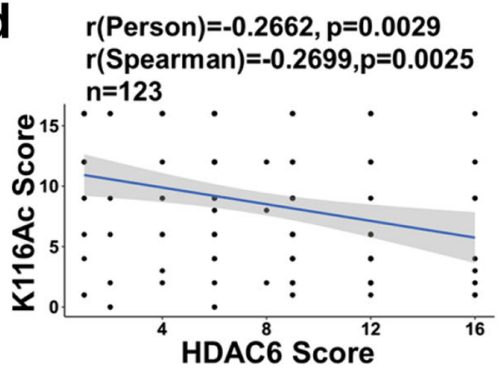

g

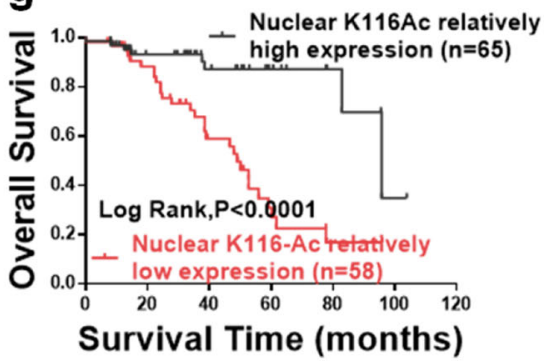

Fig. 6 Increased FRA1 protein expression and low nuclear K116 acetylation correlate with increased interleukin (IL)-6 and NANOG levels and with poor prognosis among colorectal cancer (CRC) patients. a Representative immunofluorescent images of CRC tissues co-stained with antibodies directed against FRA1 (green), STAT3pY705 (red), NANOG (carmine), and DAPI (blue). Scale bars represent $50 \mu \mathrm{m}$. b Quantification of immunohistochemically(IHC) determined FRA1 and K116Ac protein levels in nuclei and cytoplasm of cells located within CRC tissue and in normal tissue regions from CRC patients. c Representative IHC images of concurrent expression of

hetero-dimerizes with members of the JUN family, yet another level of downstream gene expression regulation $[25,30,31]$.

Acetylation is known to regulate the functional activity of TFs associated with the acquisition of CSC properties $[32,33]$. We show here that FRA1 is acetylated at a lysine residue, K116, located within its DBD. Unlike its Cterminal phosphorylation, K116 acetylation of FRA1
IL-6, FRA1, K116Ac, HDAC6, and NANOG proteins in consecutive sections of CRC tissues from two different CRC patients. Scale bars represent $50 \mu \mathrm{m}$. d Correlation analysis of HDAC6 and K116Ac protein expression levels among CRC patients. e Correlation analysis of relative mRNA expression levels of $I L-6$ and FOSL1 from GSE24551-GPL5171 dataset [26]. f, g Overall patient survival negatively correlates with high FRA1 protein level (e) and with its low nuclear K116 acetylation (f). Log-rank (Mantel-Cox) test was here employed for survival analysis

enhances its interaction with target DNA promoter sequences without affecting its stability and interaction with c-Jun. Ectopic expression of a de-acetylated (K116R) FRA1 allele results in a significant increase in self-renew and tumorigenic abilities. Although c-FOS is highly homologous to the DBD domain in FRA1, c-Fos acetylation at K148 (corresponding to K116 in FRA1) has not been reported. Moreover, knockdown of c-Fos had no evident 
a

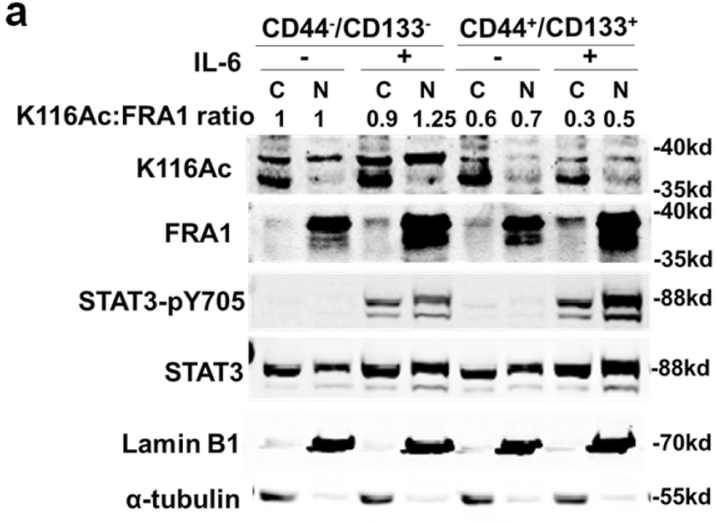

b

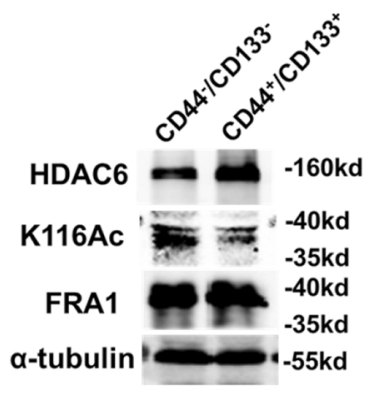

C

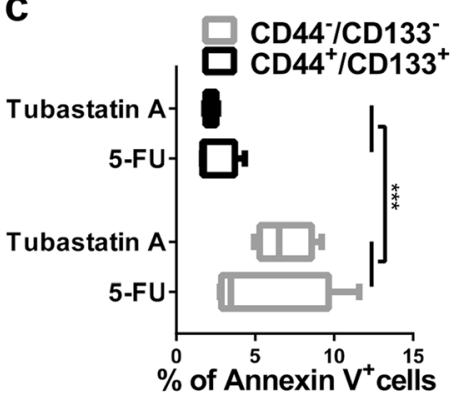

Fig. 7 Combined treatment of 5-Fluorouracil (5-FU) with the HDAC6 inhibitor Tubastatin A synergistically inhibits colorectal cancer stemlike properties and malignant growth. a Western blot analysis of nuclear and cytoplasmic extracts from sorted CD44 ${ }^{-} / \mathrm{CD} 133^{-}$and $\mathrm{CD}_{4} 4^{+} / \mathrm{CD} 133^{+}$DLD1 cells (as in Figure S1i) cultured with control or interleukin-6 supplemented medium. K116 acetylation was detected with the K116Ac antibody and normalized against the FRA1 protein. b HDAC6 and FRA1 (total and K116Ac) western blot analysis of $\mathrm{CD}_{4} 4^{-} / \mathrm{CD} 133^{-}$and $\mathrm{CD} 44^{+} / \mathrm{CD} 133^{+}$DLD1 cells sorted by

impact on the IL-6-induced EMT changes, thus indicating that c-Fos and its potential acetylation is independent of IL6/STAT3 pathway [3].

HDAC6 is a key histone deacetylase playing relevant functional roles in different cellular compartments. Although mainly localized in the cytoplasm, several lines of evidence suggest that HDAC6 can also reside in the nucleus and interact with nuclear proteins, including HDAC11; sumoylated p300; transcriptional corepressors such as ETO2 and L-CoR; and TFs such as NF-кB, Runx2, and P53 [34-36]. Here we show by western blot and IHC analysis that HDAC6 localizes to both the nucleus and cytoplasm of CRC cells and patient-derived tumor tissues. We identified HDAC6 as the enzyme responsible for FRA1 K116 deacetylation. FRA1 can physically interact with HDAC6 and this interaction is strengthened by IL-6/STAT3 signaling. Thus FRA1 regulation downstream of IL-6/STAT3 occurs at two distinct levels: through the binding of phosphorylated STAT3 to the FOSL1 promoter leading to its increased fluorescence-activated cell sorter. $\mathbf{c}$ Percentages of apoptotic (Annexin $\mathrm{V}^{+}$) cells in sorted CD44-/CD133- and CD $44^{+} / \mathrm{CD} 133^{+}$DLD1 cells when exposed to 5-FU or Tubastatin A. d Percentages of apoptotic cells in sorted CD $44^{+} / \mathrm{CD} 133^{+}$DLD1 cells when exposed to dimethyl sulfoxide (Ctrl), 5-FU, Tubastatin A, and 5-FU plus Tubastatin A. e Effects of 5-FU, Tubastatin A, and of 5-FU with Tubastatin A administered to BALB/C nude mice on day 8 when tumor had a diameter of $\sim 5 \mathrm{~mm}$ from $\mathrm{CD} 44^{+} / \mathrm{CD} 133^{+}$DLD1 cells. Histogram showing the quantification of the tumor mass

transcription, and, at the posttranslational level, through deacetylation of the lysine 116 residue, located within the DBD and resulting in enhanced interaction with the promoter of its target genes.

Among the genes known to regulate CSC properties, we used JASPAR database (http://jaspar.genereg.net/) and predicted that only NANOG, SOX2, and LGR5 encompass AP-1-binding sequences within their promoters, thus suggesting that they may represent downstream targets of the IL-6/STAT3/FRA1 signaling axis. As shown here, only $N A N O G$ does factually represent a direct FRA1 target gene. The physical interaction between FRA1 and NANOG occurs through a FRA1/AP-1-binding site (FBE) upstream of the translational start site. FRA1 deacetylation at Lys-116 enhances this interaction and further activates $N A N O G$ transcription.

It has been reported that CD24 regulates $N A N O G$ expression through STAT3 phosphorylation and that pSTAT3 (Y705) bound to the NANOG promoter drives 
tumor onset and self-renewal in the liver [12]. Moreover, it was shown that $N A N O G$ expression in CRC is regulated by extracellular insulin-like growth factor signaling via STAT3 phosphorylation [13]. Several studies have revealed the importance of NANOG as a CSC maintainer in liver cancer and CRC [10-12]. Our study showed that IL-6 treatment enhanced FOSL1 and NANOG expression in a STAT3dependent fashion. Of interest, FOSL1 gene knockout significantly blocked IL-6-induced NANOG expression, while NANOG overexpression in FOSL1 knockdown cells partially rescued the negative effects of FOSL1 ablation on chemo-resistance, self-renewal, and tumorigenesis. Notably, NANOG knockdown significantly inhibited IL-6 induced in vitro ability of migration, invasion, chemo-resistance, and self-renew and in vivo ability of tumorigenesis. Hence, NANOG represents, presumably together with others [18], a bona fide downstream effector of FRA1, rather than STAT3, in the regulation of CSC properties downstream of IL-6. In human ESCs, transforming growth factor- $\beta /$ Activin-responsive SMADs bind to the NANOG promoter and play an essential role in sustaining self-renewal [37]. We showed here that, in the context of inflammation and CRC, the regulation of the NANOG stem-like pathway was hijacked by the inflammatory IL-6/STAT3/FRA1 pathway. These results were validated in a large $(n=123)$ cohort of prospectively collected human colorectal carcinomas. We found that, while FRA1 was mainly localized in the nucleus of cancer cells, its acetylated form was mainly present in the cytoplasm, i.e., indicative of low K116Ac levels in its nuclear fraction. More importantly, high FRA1 protein expression and its relatively low K116 acetylation levels in the nucleus highly correlate with IL- 6 secretion and with poor prognosis and overall survival among CRC patients. In summary, the results of western blots and IHC both showed that IL-6 deacetylates FRA1-K116 in the CRC cells.

The elucidation of the cellular and molecular mechanisms underlying the enhancement of colon cancer stemness downstream of the IL-6/STAT3/FRA1 signaling axis opens novel opportunities to develop alternative and possibly more effective therapeutic strategies. Small molecules inhibiting lysine acetylation (HDAC inhibitors) have been reported in the scientific literature, some of which with Food andDrug Administrationapproval, for the treatment of a broad spectrum of cancer-related pathologies. SAHA (also known as Vorinostat) was approved to treat cutaneous $\mathrm{T}$ cell lymphoma [20]. The antiepileptic drug valproic acid also inhibits HDACs and is currently being employed in several clinical trials for various indications [20]. Tubastatin A is a neuroprotective and specific inhibitor of HDAC6 [38]. HDAC6 maintains glioma stem cells (GSCs) through GLI1 signaling and its specific inhibition, when combined with X-ray irradiation, suppresses the tumorigenic capacity of GSCs in vivo [39].
5-FU represents one of the most common chemotherapeutic drugs in the treatment of CRC. However, its curative effects as a single agent are far from optimal. As observed in this study and supported by several previous reports, the stem-like $\mathrm{CD} 44^{+} / \mathrm{CD} 133^{+}$colon cancer cells are chemo-resistant, a characteristic that is enhanced by IL6 stimulation and the subsequent FRA1 deacetylation. Here we showed that the combination of 5-FU with the HDAC6 inhibitor Tubastatin A, designed to target both the more committed CRC cells (by 5-FU) and the small but chemoresistant CSC fraction (by Tubastatin A), dramatically inhibited tumor growth in vivo with no significant in vivo toxicity. Hence, the combined treatment of a chemotherapeutic agent with an HDAC6 inhibitor may offer an improved and novel curative approach for CRC patients, especially in those cases where the IL-6/STAT3/ FRA1 signaling axis is activated.

In conclusion, upon inflammation and IL-6 secretion from the microenvironment, the STAT3 pathway becomes activated and promotes CRC stemness and malignancy through the transcriptional (promoter binding) and posttranslational (K116 deacetylation) upregulation of FOSL1/ FRA1. HDAC6 is responsible for the deacetylation of the Lys-116 residue in FRA1. NANOG is a direct FRA1 target gene likely to play a central role in conferring increased stemness and malignancy to the cancer cell. Last, combinatorial treatment with a conventional cytotoxic agent (5FU) and an HDAC6 inhibitor (Tubastatin A) may offer novel potential therapeutic options for CRC patients.

\section{Materials and methods}

\section{Patient samples}

A total of 123 human CRC samples were collected at the Second Affiliated Hospital of Zhejiang University School of Medicine after informed consent had been given by all patients. The clinicopathological characteristics of the clinical specimens are summarized in Supplementary Table S2.

\section{Statistical analysis}

Data represent the mean \pm SD of at least three independent experiments. Animal studies were performed blinded. No animals were excluded from study results. The animal experimental groups were blinded to the experimentalists during the experiment and data collection. BALB/C nude mice (male, 5 weeks of age) were selected for each group in a randomized fashion. The Log-rank (Mantel-Cox) test was used for survival analysis. The two-tailed $t$ test was used to evaluate statistical differences in different groups. Pearson 
correlation and Spearman rank correlation tests were computed to assess the relativity of two groups. All analyses were performed using the GraphPad Prism6 software and R x64 3.4.4 statistical software. ${ }^{\#, *} p<0.05$; ${ }^{\#, * * p} p<0.01$; $\# \# \#, * * * p<0.001$.

\section{Study approval}

All animal studies were reviewed and approved by the Laboratory Animal Welfare Committee of Zhejiang University (approval number ZJU2015-282-01). The procedures related to human subjects were approved by the Medical Ethics Committee of Zhejiang University School of Medicine (approval number 2015-013).

See Online Supplementary Material and Methods for additional experimental procedures [40-49].

Acknowledgements The authors are grateful to the contribution of the specimen donors. We thank Professor Xin-Hua Feng from Life Sciences Institute and Innovation Center for Cell Signaling Network, Zhejiang University for providing HDAC6 overexpressing plasmids; Professor Qun-Ying Lei from Key Laboratory of Metabolism and Molecular Medicine, Ministry of Education, Fudan University for providing HDAC1-6 overexpressing plasmids; Professor Cheng Qian from the Institute of Pathology and Southwest Cancer Center, Southwest Hospital, Third Military Medical University for providing $\mathrm{Lv}-N A N O G$, Lv-shNANOG, and Lv-scrambled plasmids; and Professor Chong Liu from the Department of Pathology \& Pathophysiology, Zhejiang University School of Medicine for help with immunofluorescence technology. This work was supported by National Natural Science Foundation of China (81572384, 81372138, 81702401 and 81771518), National Key R\&D Program of China (2016YFC1303401), and National Science and Technology Major Project of China (2018ZX10302206-006-007). RF is supported by ZJU funds and by the Dutch Digestive Foundation (FP 15-09), Dutch Cancer Society, and the Erasmus MC (EMCR 2015-8090).

\section{Compliance with ethical standards}

Conflict of interest The authors declare that they have no conflict of interest.

Publisher's note: Springer Nature remains neutral with regard to jurisdictional claims in published maps and institutional affiliations.

Open Access This article is licensed under a Creative Commons Attribution 4.0 International License, which permits use, sharing, adaptation, distribution and reproduction in any medium or format, as long as you give appropriate credit to the original author(s) and the source, provide a link to the Creative Commons license, and indicate if changes were made. The images or other third party material in this article are included in the article's Creative Commons license, unless indicated otherwise in a credit line to the material. If material is not included in the article's Creative Commons license and your intended use is not permitted by statutory regulation or exceeds the permitted use, you will need to obtain permission directly from the copyright holder. To view a copy of this license, visit http://creativecommons. org/licenses/by/4.0/.

\section{References}

1. Hanahan D, Weinberg RA. Hallmarks of cancer: the next generation. Cell. 2011;144:646-74.

2. Lasry A, Zinger A, Ben-Neriah Y. Inflammatory networks underlying colorectal cancer. Nat Immunol. 2016;17:230-40.

3. Liu H, Ren G, Wang T, Chen Y, Gong C, Bai Y, et al. Aberrantly expressed Fra-1 by IL-6/STAT3 transactivation promotes colorectal cancer aggressiveness through epithelial-mesenchymal transition. Carcinogenesis. 2015;36:459-68.

4. Grivennikov SI, Karin M. Inflammatory cytokines in cancer: tumour necrosis factor and interleukin 6 take the stage. Ann Rheum Dis. 2011;1:i104-i108.

5. Wan S, Zhao E, Kryczek I, Vatan L, Sadovskaya A, Ludema G, et al. Tumor-associated macrophages produce interleukin 6 and signal via STAT3 to promote expansion of human hepatocellular carcinoma stem cells. Gastroenterology. 2014;147:1393-404.

6. Du L, Wang H, He L, Zhang J, Ni B, Wang X, et al. CD44 is of functional importance for colorectal cancer stem cells. Clin Cancer Res. 2008;14:6751-60.

7. Wang D, Fu L, Sun H, Guo L, DuBois RN. Prostaglandin E2 promotes colorectal cancer stem cell expansion and metastasis in mice. Gastroenterology. 2015;149:1884-95.

8. Dalerba P, Dylla SJ, Park IK, Liu R, Wang X, Cho RW, et al. Phenotypic characterization of human colorectal cancer stem cells. Proc Natl Acad Sci USA. 2007;104:10158-63.

9. Ricci-Vitiani L, Lombardi DG, Pilozzi E, Biffoni M, Todaro M, Peschle $\mathrm{C}$, et al. Identification and expansion of human coloncancer-initiating cells. Nature. 2007;445:111-5.

10. Zhang J, Espinoza LA, Kinders RJ, Lawrence SM, Pfister TD, Zhou M, et al. NANOG modulates stemness in human colorectal cancer. Oncogene. 2013;32:4397-405.

11. Ibrahim EE, Babaei-Jadidi R, Saadeddin A, Spencer-Dene B, Hossaini S, Abuzinadah M, et al. Embryonic NANOG activity defines colorectal cancer stem cells and modulates through AP1and TCF-dependent mechanisms. Stem Cells. 2012;30:2076-87.

12. Lee TK, Castilho A, Cheung VC, Tang KH, Ma S, Ng IO. CD24 $(+)$ liver tumor-initiating cells drive self-renewal and tumor initiation through STAT3-mediated NANOG regulation. Cell Stem Cell. 2011;9:50-63.

13. Yao C, Su L, Shan J, Zhu C, Liu L, Liu C, et al. IGF/STAT3/ NANOG/Slug signaling axis simultaneously controls epithelialmesenchymal transition and stemness maintenance in colorectal cancer. Stem Cells. 2016;34:820-31.

14. Kakumoto K, Sasai K, Sukezane T, Oneyama C, Ishimaru S, Shibutani K, et al. FRA1 is a determinant for the difference in RAS-induced transformation between human and rat fibroblasts. Proc Natl Acad Sci USA. 2006;103:5490-5.

15. Sayan AE, Stanford R, Vickery R, Grigorenko E, Diesch J, Kulbicki K, et al. Fra-1 controls motility of bladder cancer cells via transcriptional upregulation of the receptor tyrosine kinase AXL. Oncogene. 2012;31:1493-503.

16. Hong A, Moriceau G, Sun L, Lomeli S, Piva M, Damoiseaux R, et al. Exploiting drug addiction mechanisms to select against MAPKi-resistant melanoma. Cancer Discov. 2018;8:74-93.

17. Tam WL, Lu H, Buikhuisen J, Soh BS, Lim E, Reinhardt F, et al. Protein kinase $\mathrm{C} \alpha$ is a central signaling node and therapeutic target for breast cancer stem cells. Cancer Cell. 2013;24:347-64.

18. Lau EY, Lo J, Cheng BY, Ma MK, Lee JM, Ng JK, et al. Cancerassociated fibroblasts regulate tumor-initiating cell plasticity in hepatocellular carcinoma through c-Met/FRA1/HEY1 signaling. Cell Rep. 2016;15:1175-89.

19. Belguise K, Milord S, Galtier F, Moquet-Torcy G, Piechaczyk M, Chalbos D. The PKC $\theta$ pathway participates in the aberrant 
accumulation of Fra-1 protein in invasive ER-negative breast cancer cells. Oncogene. 2012;31:4889-97.

20. Ali I, Conrad RJ, Verdin E, Ott M. Lysine acetylation goes global: from epigenetics to metabolism and therapeutics. Chem Rev. 2018;118:1216-52.

21. Wang D, Kon N, Lasso G, Jiang L, Leng W, Zhu WG, et al. Acetylation-regulated interaction between p53 and SET reveals a widespread regulatory mode. Nature. 2016;538:118-22.

22. Vries RG, Prudenziati M, Zwartjes C, Verlaan M, Kalkhoven E, Zantema A. A specific lysine in c-Jun is required for transcriptional repression by E1A and is acetylated by p300. EMBO J. 2001;20:6095-103.

23. Canettieri G, Di Marcotullio L, Greco A, Coni S, Antonucci L, Infante P, et al. Histone deacetylase and Cullin3-REN(KCTD11) ubiquitin ligase interplay regulates Hedgehog signalling through Gli acetylation. Nat Cell Biol. 2010;12:132-42.

24. Ting H, Guangyong Z, Pingyu Z, Jia J, Jing L. LAceP: lysine acetylation site prediction using logistic regression classifiers. PLoS ONE. 2014;9:e89575.

25. Pakay JL, Diesch J, Gilan O, Yip YY, Sayan E, Kolch W, et al. A 19S proteasomal subunit cooperates with an ERK MAPKregulated degron to regulate accumulation of Fra-1 in tumour cells. Oncogene. 2012;31:1817-24.

26. Sveen A, Agesen TH, Nesbakken A, Rognum TO, Lothe RA, Skotheim RI. Transcriptome instability in colorectal cancer identified by exon microarray analyses: associations with splicing factor expression levels and patient survival. Genome Med. 2011;3:32.

27. van der Zee M, Sacchetti A, Cansoy M, Joosten R, Teeuwssen M, Heijmans-Antonissen C, et al. IL6/JAK1/STAT3 signaling blockade in endometrial cancer affects the ALDHhi/CD126+ stem-like component and reduces tumor burden. Cancer Res. 2015;75:3608-22.

28. He W, Wu J, Shi J, Huo YM, Dai W, Geng J, et al. IL22RA1/ STAT3 signaling promotes stemness and tumorigenicity in pancreatic cancer. Cancer Res. 2018;78:3293-305.

29. Kryczek I, Lin Y, Nagarsheth N, Peng D, Zhao L, Zhao E, et al. IL-22( +$) \mathrm{CD} 4(+)$ T cells promote colorectal cancer stemness via STAT3 transcription factor activation and induction of the methyltransferase DOT1L. Immunity. 2014;40:772-84.

30. Wang Q, Ni H, Lan L, Wei X, Xiang R, Wang Y. Fra-1 protooncogene regulates IL-6 expression in macrophages and promotes the generation of M2d macrophages. Cell Res. 2010;20:701-12.

31. Zerbini LF, Wang Y, Cho JY, Libermann TA. Constitutive activation of nuclear factor kappaB p50/p65 and Fra-1 and JunD is essential for deregulated interleukin 6 expression in prostate cancer. Cancer Res. 2003;63:2206-15.

32. Qi J, Singh S, Hua WK, Cai Q, Chao SW, Li L, et al. HDAC8 inhibition specifically targets Inv (16) acute myeloid leukemic stem cells by restoring p53 acetylation. Cell Stem Cell. 2015;17:597-610.

33. Yoon DS, Choi Y, Jang Y, Lee M, Choi WJ, Kim SH, et al. SIRT1 directly regulates SOX2 to maintain self-renewal and multipotency in bone marrow-derived mesenchymal stem cells. Stem Cells. 2014;32:3219-31.

34. Yang XJ, Grégoire S. Class II histone deacetylases: from sequence to function, regulation, and clinical implication. Mol Cell Biol. $2005 ; 25: 2873-84$.
35. Liu Y, Peng L, Seto E, Huang S, Qiu Y. Modulation of histone deacetylase 6 (HDAC6) nuclear import and tubulin deacetylase activity through acetylation. J Biol Chem. 2012;287:29168-74.

36. Ryu HW, Shin DH, Lee DH, Choi J, Han G, Lee KY, et al. HDAC6 deacetylates p53 at lysines $381 / 382$ and differentially coordinates p53-induced apoptosis. Cancer Lett. 2017;391: $162-71$.

37. Xu RH, Sampsell-Barron TL, Gu F, Root S, Peck RM, Pan G, et al. NANOG is a direct target of TGF-beta/activin-mediated SMAD signaling in human ESCs. Cell Stem Cell. 2008;3:196206.

38. Butler KV, Kalin J, Brochier C, Vistoli G, Langley B, Kozikowski AP. Rational design and simple chemistry yield a superior, neuroprotective HDAC6 inhibitor, tubastatin A. J Am Chem Soc. 2010;132:10842-6.

39. Yang W, Liu Y, Gao R, Yu H, Sun T. HDAC6 inhibition induces glioma stem cells differentiation and enhances cellular radiation sensitivity through the SHH/Gli1 signaling pathway. Cancer Lett. 2018;415:164-76.

40. Glover JN, Harrison SC. Crystal structure of the heterodimeric bZIP transcription factor c-Fos-c-Jun bound to DNA. Nature. 1995;373:257-61.

41. Chen L, Glover JN, Hogan PG, Rao A, Harrison SC. Structure of the DNA-binding domains from NFAT, Fos and Jun bound specifically to DNA. Nature. 1998;392:42-48.

42. Jacobson MP, Pincus DL, Rapp CS, Day TJ, Honig B, Shaw DE, et al. A hierarchical approach to all-atom protein loop prediction. Proteins. 2004;55:351-67.

43. Maier JA, Martinez C, Kasavajhala K, Wickstrom L, Hauser KE, Simmerling C. ff14SB: improving the accuracy of protein side chain and backbone parameters from ff99SB. J Chem Theory Comput. 2015;11:3696-713.

44. Salomon-Ferrer R, Case DA, Walker RC. An overview of the Amber biomolecular simulation package. Wiley Interdiscip RevComput Mol Sci. 2013;3:198-210.

45. Hou T, Wang J, Li Y, Wang W. Assessing the performance of the MM/PBSA and MM/GBSA methods. 1. The accuracy of binding free energy calculations based on molecular dynamics simulations. J Chem Inf Model. 2011;51:69-82.

46. Xu L, Sun H, Li Y, Wang J, Hou T. Assessing the performance of MM/PBSA and MM/GBSA methods. 3. The impact of force fields and ligand charge models. J Phys Chem B. 2013; 117:8408-21.

47. Sun H, Li Y, Shen M, Tian S, Xu L, Pan P, et al. Assessing the performance of MM/PBSA and MM/GBSA methods. 5. Improved docking performance using high solute dielectric constant MM/ GBSA and MM/PBSA rescoring. Phys Chem Chem Phys. 2014;16:22035-45.

48. Kollman PA, Massova I, Reyes C, Kuhn B, Hu S, Chong L, et al. Calculating structures and free energies of complex molecules: combining molecular mechanics and continuum models. Acc Chem Res. 2000;33:889-97.

49. Hou T, Li N, Li Y, Wang W. Characterization of domain-peptide interaction interface: prediction of SH3 domain-mediated proteinprotein interaction network in yeast by generic structure-based models. J Proteome Res. 2012;11:2982-95. 